\title{
A GERSTEN-WITT SPECTRAL SEQUENCE FOR REGULAR SCHEMES
}

\author{
By PAUl BALMER AND CHARles WALTER
}

ABStRACT. - A spectral sequence is constructed whose non-zero $E_{1}$-terms are the Witt groups of the residue fields of a regular scheme $X$, arranged in Gersten-Witt complexes, and whose limit is the four global Witt groups of $X$. This has several immediate consequences concerning purity for Witt groups of low-dimensional schemes. We also obtain an easy proof of the Gersten Conjecture in dimension smaller than 5. The Witt groups of punctured spectra of regular local rings are also computed.

(c) 2002 Éditions scientifiques et médicales Elsevier SAS

RÉSUMÉ. - Nous construisons une suite spectrale dont les termes $E_{1}$ non nuls sont les groupes de Witt des corps résiduels d'un schéma régulier $X$, organisés en complexes de Gersten-Witt. La suite spectrale converge vers les quatre groupes de Witt globaux de $X$. Ceci a plusieurs conséquences immédiates sur la pureté des groupes de Witt de schémas de basse dimension. Nous obtenons également une preuve facile de la Conjecture de Gersten en dimension inférieure à 5 . Nous calculons aussi les groupes de Witt des spectres épointés d'anneaux locaux réguliers.

(C) 2002 Éditions scientifiques et médicales Elsevier SAS

Let $X$ be a regular integral separated noetherian scheme of finite Krull dimension in which 2 is everywhere invertible. (We will maintain these hypotheses throughout the introduction.) The Witt groups of the residue fields of $X$ are known to form a cochain complex

$$
\mathcal{W}_{X}: 0 \rightarrow W(K) \rightarrow \bigoplus_{x_{1} \in X^{(1)}} W\left(k\left(x_{1}\right)\right) \rightarrow \cdots \rightarrow \bigoplus_{x_{e} \in X^{(e)}} W\left(k\left(x_{e}\right)\right) \rightarrow 0
$$

called a Gersten-Witt complex; here $X^{(p)}$ denotes the set of points of codimension $p$ in $X$, while $K$ is the field of rational functions on $X$, and $e=\operatorname{dim}(X)$. This Gersten-Witt complex is only the tip of an iceberg; below the water level lurks a coniveau spectral sequence, similar to the Gersten spectral sequence in $K$-theory, but having a rather special form.

THEOREM (Gersten-Witt spectral sequence). - There exists a spectral sequence converging to $E^{n}=W^{n}(X)$ such that $E_{1}^{p q}=0$ unless $0 \leqslant p \leqslant \operatorname{dim}(X)$, and such that the horizontal line

$$
0 \rightarrow E_{1}^{0 q} \stackrel{d_{1}}{\longrightarrow} E_{1}^{1 q} \stackrel{d_{1}}{\longrightarrow} E_{1}^{2 q} \stackrel{d_{1}}{\longrightarrow} \cdots \stackrel{d_{1}}{\longrightarrow} E_{1}^{\operatorname{dim}(X), q} \rightarrow 0
$$

of the spectral sequence vanishes for $q \not \equiv 0(\bmod 4)$, while the line is the Gersten-Witt complex for all $q \equiv 0(\bmod 4)$.

This is a slightly simplified version of our main Theorem 7.2. 
The Witt groups $W^{n}(X)$ identified by the theorem as the limit of the spectral sequence are the derived Witt groups of Balmer [1-3]. Briefly, these groups classify pairs $(\mathcal{E}, \phi)$ where $\mathcal{E}$ is a bounded complex of vector bundles on $X$, where $\mathcal{E}^{*}[n]$ is the shift by $n$ of the dual complex, and where $\phi: \mathcal{E} \rightarrow \mathcal{E}^{*}[n]$ is a symmetric isomorphism in the derived category. The group $W^{n}(X)$ classifies such $(\mathcal{E}, \phi)$ up to isometry and modulo the metabolic classes, i.e. modulo those $(\mathcal{E}, \phi)$ which admit a totally isotropic 'sub'-complex which divides them evenly in two. The groups $W^{n}(X)$ depend only on $n$ modulo 4 . Moreover, the even derived Witt groups have been identified via

$$
W^{4 \ell}(X) \cong W(X), \quad W^{4 \ell+2}(X) \cong W^{-}(X),
$$

as the usual Witt groups of symmetric and skew-symmetric vector bundles on $X$ (Balmer [3, Theorem 4.7]), while the odd derived Witt groups $W^{4 \ell+1}(X)$ and $W^{4 \ell+3}(X)$ are isomorphic to the Witt groups of formations described in Fernández-Carmena [9], Pardon [18], and Ranicki [23,22] (see Walter [27]).

The periodicity of the Gersten-Witt spectral sequence and the fact that three out of every four lines vanish allow us to give quick proofs of a number of results concerning Witt groups of low-dimensional schemes. The following one is part of Theorem 10.1.

THEOREM (Low dimensions). - Suppose that $\operatorname{dim}(X) \leqslant 7$. Then the derived Witt groups $W^{n}:=W^{n}(X)$ and the Witt cohomology groups $H^{n}(\mathcal{W}):=H^{n}\left(\mathcal{W}_{X}\right)$ fit into the following exact sequence:

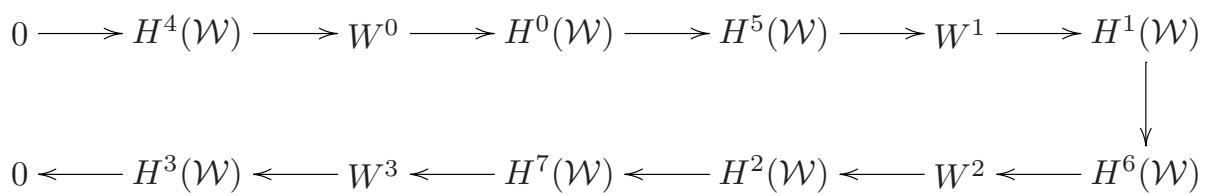

We also establish that the first differential $d_{1}^{00}: \mathcal{W}_{X}^{0} \rightarrow \mathcal{W}_{X}^{1}$ in our Gersten-Witt complex is the classical residue map, so our $H^{0}\left(\mathcal{W}_{X}\right)$ is the unramified Witt group $W_{\mathrm{nr}}(X)$. The next two results (Corollaries 10.2 and 10.3) then follow without any additional work.

THEOREM (Weak purity in dimension 4 ). - If $\operatorname{dim}(X) \leqslant 4$, then the natural map $W(X) \rightarrow W_{\mathrm{nr}}(X)$ is surjective.

THEOREM (Purity in dimension 3 ). - If $\operatorname{dim}(X) \leqslant 3$, then the natural map $W(X) \rightarrow W_{\mathrm{nr}}(X)$ is an isomorphism.

We can also use the spectral sequence to prove the Gersten Conjecture for Witt groups of any regular local ring of dimension up to 4 , even a regular local ring which does not contain a field! For this we augment the Gersten-Witt complex of a scheme $X$ by adding the natural homomorphism $W(X) \rightarrow W(K)$ at the beginning.

$$
0 \rightarrow W(X) \rightarrow W(K) \rightarrow \bigoplus_{x_{1} \in X^{(1)}} W\left(k\left(x_{1}\right)\right) \rightarrow \cdots \rightarrow \bigoplus_{x_{e} \in X^{(e)}} W\left(k\left(x_{e}\right)\right) \rightarrow 0 .
$$

The following result is Corollary 10.4.

THEOREM (Gersten conjecture in dimension $\leqslant 4)$. - Let $X=\operatorname{Spec}(R)$ with $R$ a regular local ring of Krull dimension $e \leqslant 4$ and containing $\frac{1}{2}$. Then the augmented Gersten-Witt complex (4) is exact. 
The above results include and improve most of the known Gersten-Witt related results in low dimension. Nevertheless, they are all immediate no-work consequences of the existence and form of the Gersten-Witt spectral sequence and of the identification of certain components of the $d_{1}$ coboundaries with classical residue maps. Morever, the periodicity and vanishing in the GerstenWitt spectral sequence gives the first indication of a reason behind the way that low-dimension Gersten-Witt results start to get more complicated beginning in dimension 4. Our construction of the spectral sequence also gives a new construction of the Gersten-Witt complex, and it is high time that at least one construction of that complex gets into print.

All the results of this paper can and will be proven for twisted Witt groups $W^{n}(X, L)$. Twisting the duality by a line bundle $L$ changes the global Witt groups, and it changes the differentials (but not the groups) of the Gersten-Witt complex. These twists are useful for applications in algebraic geometry and are indispensable for defining transfer maps for Witt groups of projective varieties.

Purity was known for Witt groups of affine regular schemes of dimension up to 3 (Parimala [20], Ojanguren et al. [16]), but the global result (for any regular integral noetherian separated scheme of dimension $\leqslant 3$ with 2 everywhere invertible) is new, as is weak purity in dimension 4 . Ojanguren and Panin [17] have established purity for Witt groups of regular local rings of any dimension which contain a field of characteristic $\neq 2$. As far as we know, the 'plain' Gersten Conjecture up to dimension 4 is totally new. We have been informed that Barge, Sansuc, and Vogel worked on both the Gersten-Witt complex and a spectral sequence several years ago although no paper ever appeared, while Pardon has recently informed us that he has some new results [19] related to the complex, some of which overlap with this paper.

\section{The Gersten-Witt complex}

For discrete valuation rings and Dedekind domains, the Gersten-Witt complex reduces to a single map $W(K) \rightarrow \bigoplus W(k(x))$, the classical (second) residue, which has been around for quite a while (cf. Milnor-Husemoller [14, Chapter IV]). More recently, a Gersten-Witt complex in higher dimension has been constructed for schemes of finite type over a ground field in unpublished work by Schmid [25], Ettner [8], Rost [24]. Pardon's new paper [19] also contains a Gersten-Witt complex for regular schemes of finite type over a field.

This paper produces the Gersten-Witt complex as the $E_{1}$ term of the spectral sequence. One complication, however, is that the identification of the groups in the Gersten-Witt complex as $\bigoplus W\left(k\left(x_{p}\right)\right)$ is not entirely canonical but rather requires one to choose a system of isomorphisms between one-dimensional vector spaces $\operatorname{Ext}_{\mathcal{O}_{X, x_{p}}}^{p}\left(k\left(x_{p}\right), \mathcal{O}_{X, x_{p}}\right) \cong k\left(x_{p}\right)$. This is because in our approach the groups written as $W\left(k\left(x_{p}\right)\right)$ that appear in the Gersten-Witt complex or spectral sequence are really Witt groups of finite-length modules over the local ring $\mathcal{O}_{X, x_{p}}$ (Proposition 6.2) for the duality functor $\operatorname{Ext}_{\mathcal{O}_{X, x_{p}}}^{p}\left(-, \mathcal{O}_{X, x_{p}}\right)$. Thus the Gersten-Witt complex for $X$ (with the trivial twist $\mathcal{O}_{X}$ ) is 'canonically'

$$
0 \rightarrow \bigoplus_{x_{0} \in X^{(0)}} \widetilde{W}\left(x_{0}\right) \rightarrow \bigoplus_{x_{1} \in X^{(1)}} \widetilde{W}\left(x_{1}\right) \rightarrow \cdots \rightarrow \bigoplus_{x_{e} \in X^{(e)}} \widetilde{W}\left(x_{e}\right) \rightarrow 0
$$

where $e=\operatorname{dim}(X)$, where the coboundaries are as in Proposition 8.5, and where each $\widetilde{W}\left(x_{p}\right):=W\left(k\left(x_{p}\right), \omega_{x_{p} / X}\right)$ is the Witt group of the residue field $k\left(x_{p}\right)$ twisted by the onedimensional vector space

$$
\omega_{x_{p} / X}=\operatorname{Ext}_{\mathcal{O}_{X, x_{p}}}^{p}\left(k\left(x_{p}\right), \mathcal{O}_{X, x_{p}}\right) \cong \Lambda^{p}\left(\mathfrak{m}_{x_{p}} / \mathfrak{m}_{x_{p}}^{2}\right)^{*} .
$$

If there is a global twist $L$, then the 'canonical' local coefficients have a further twist by $L$. Every single Witt group of a field in this paper is 'canonically' one of these twisted Witt groups, except 
briefly in the proof of Proposition 9.2(c). However, we do not wish to burden the reader with complicated notations, so we will write $W(k(x))$.

The strategy of the article is not very complicated. It relies on the triangular Witt groups of [1-3], which we discuss in Sections 1 and 2. In those papers the Witt groups are presented as invariants of triangulated categories with duality, and a 'short exact sequence' $\mathbf{D} \hookrightarrow \mathbf{C} \rightarrow \mathbf{C} / \mathbf{D}$ of triangulated categories with duality yields a 'localization' long exact sequence of derived Witt groups. Confronted with a filtration of a triangulated category with duality, we can assemble the localization exact sequences into a spectral sequence using an exact couple. This is done in Section 3.

The idea is to apply this construction to the coniveau filtration of the derived category of $X$. The categorical generalities give immediately the existence of some spectral sequence, periodic in $q$ and converging to the derived Witt groups of $X$. The problem is to identify the terms $E_{1}^{p q}$ with either the Witt groups of the residue fields of $X$ or with 0 . This dévissage is the technical core of the article and is developed in Sections 4, 5 and 6. The pieces fall into place in Section 7, where we establish the main Theorem 7.2. Although this type of local computation is not terribly new or surprising, its coalescence with the derived-category approach to Witt groups is fertile and is the real point of this article.

In Section 8 we include more details on the differentials appearing in our Gersten-Witt complex. For the moment we do not know whether our differentials are the same as those of Schmid [25] and Pardon [19], although we hope to treat this problem in our next paper.

The article ends with several applications of the results of the central sections. The first is the computation of the Witt groups of the punctured spectrum of a regular local ring (Theorem 9.1). This was described in the Introduction of [2] as one of the motivations for the derived-category approach to Witt groups. It requires dévissage but not the spectral sequence. In Section 10, the short proofs of global purity for Witt groups in dimension 3, of weak purity in dimension 4 and of the Gersten Conjecture in dimension $\leqslant 4$ illustrate the quiet power of the spectral sequence.

This paper is part of an ongoing project on derived Witt groups whose next installment [4] should include among other things a Gersten-Witt spectral sequence for Witt groups of coherent sheaves on singular schemes.

\section{Review of triangular Witt groups}

We will recall a number of definitions and results from Balmer [1,2]. We begin by fixing some basic terminology for categories, and then we move on to dualities on triangulated category and to triangular Witt groups.

We will say that an additive category contains $\frac{1}{2}$ if its Hom groups are uniquely 2-divisible, or equivalently if the category is $\mathbb{Z}\left[\frac{1}{2}\right]$-linear.

We will use the following definition of exact functors between triangulated categories. It is more restrictive than the official definition (which only assumes that the functor commutes with the translation up to a specified natural isomorphism), but it suffices for this paper. Let $\mathbf{C}$ and $\mathbf{D}$ be triangulated categories, and let $\delta= \pm 1$. We will say that a covariant functor $F: \mathbf{C} \rightarrow \mathbf{D}$ is $\delta$-exact if it is additive, commutes with the translations $T_{\mathbf{D}} F=F T_{\mathbf{C}}$, and if for every exact triangle in $\mathbf{C}$

$$
X \stackrel{u}{\longrightarrow} Y \stackrel{v}{\longrightarrow} Z \stackrel{w}{\longrightarrow} T_{\mathbf{C}} X
$$

the triangle

$$
F X \stackrel{F u}{\longrightarrow} F Y \stackrel{F v}{\longrightarrow} F Z \stackrel{\delta \cdot F w}{\longrightarrow} T_{\mathbf{D}} F X
$$


is exact in $\mathbf{D}$. An exact functor is a functor which is either $(+1)$-exact or $(-1)$-exact. A morphism of exact functors is a morphism of functors $\alpha: F \rightarrow G$ such that $T \alpha=\alpha T$ if $F$ and $G$ are of the same parity, but such that $T \alpha=-\alpha T$ if $F$ and $G$ are of the opposite parity.

A contravariant functor $G: \mathbf{C}^{\mathrm{op}} \rightarrow \mathbf{D}$ is $\delta$-exact if it is additive, commutes with the translations $T_{\mathbf{D}} G=G T_{\mathbf{C}}^{-1}$, and if for every exact triangle (7) in $\mathbf{C}$ the following triangle is exact in $\mathbf{D}$

$$
G Z \stackrel{G v}{\longrightarrow} G Y \stackrel{G u}{\longrightarrow} G X \stackrel{\delta \cdot T_{\mathbf{D}} G w}{\longrightarrow} T_{\mathbf{D}} G Z .
$$

Definition 1.1. - A duality on a triangulated category $\mathbf{C}$ is a triple $\left({ }^{*}, \delta, \varpi\right)$ with $\delta= \pm 1$, with ${ }^{*}: \mathbf{C}^{\text {op }} \rightarrow \mathbf{C}$ a $\delta$-exact functor, and with $\varpi: 1 \stackrel{\sim}{\longrightarrow}{ }^{* *}$ an isomorphism of functors such that $T \varpi=\varpi T$ and such that for all objects $X$ the maps $\varpi_{X^{*}}: X^{*} \rightarrow X^{* * *}$ and $\varpi_{X}^{*}: X^{* * *} \rightarrow X^{*}$ are inverse isomorphisms.

A duality $\left({ }^{*}, \delta, \varpi\right)$ induces shifted dualities $\left(D_{n}, \delta_{n}, \varpi_{n}\right)$ for all $n \in \mathbb{Z}$ by

$$
D_{n}:=T^{n} \circ^{*}, \quad \delta_{n}:=(-1)^{n} \delta, \quad \varpi_{n}:=(-1)^{n(n+1) / 2} \delta^{n} \varpi .
$$

(The point of these signs is the recurrence relations $\delta_{n}=-\delta_{n-1}$ and $\varpi_{n}=\delta_{n} \varpi_{n-1}$.) Fixing an $n$, let $B^{\#}=T^{n}\left(B^{*}\right)=D_{n}(B)$. A map of the form $u: A \rightarrow B^{\#}$ has a transpose $u^{\mathrm{t}}: B \rightarrow A^{\#}$ given by $u^{\mathrm{t}}:=u^{\#} \circ \varpi_{n, B}$. Morphisms of the form $v: A^{\#} \rightarrow B$ also have transposes. One has $u^{\text {tt }}=u$ for all $u$. A morphism $w: A \rightarrow A^{\#}$ is symmetric if $w^{\mathrm{t}}=w$. A symmetric object of $\mathbf{C}$ with respect to the shifted duality is a pair $(A, w)$ with $w: A \stackrel{\sim}{\longrightarrow} A^{\#}$ a symmetric isomorphism. Two symmetric objects $(A, w)$ and $(B, s)$ are isometric if there exists an isomorphism $r: A \stackrel{\sim}{\longrightarrow} B$ such that $w=r^{\#} s r$.

Proposition 1.2 (Balmer [2, Theorem 2.6]). - Let $\left({ }^{*}, \delta, \varpi\right)$ be a duality on a triangulated category $\mathbf{C}$ containing $\frac{1}{2}$, and let $\left(\#, \delta_{n}, \varpi_{n}\right)$ be the nth shifted duality. If

$$
u: A \rightarrow T^{-1}\left(A^{\#}\right)=(T A)^{\#}
$$

is a map which is symmetric with respect to the $(n-1)$-st shifted duality, then there exists a commutative diagram with exact rows

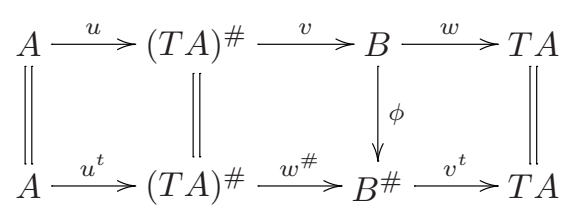

and with $\phi$ a symmetric isomorphism with respect to \#. Moreover, $(B, \phi)$ is uniquely determined by $(A, u)$ up to isometry.

In the situation of Proposition 1.2 we write $\operatorname{Cone}(A, u):=(B, \phi)$. A symmetric object is metabolic if it is isometric to a $\operatorname{Cone}(A, u)$. The object $(T A)^{\#}$ is a Lagrangian of $(B, \phi)$. Two symmetric objects $(E, \sigma)$ and $(F, \tau)$ are Witt-equivalent if there exist metabolic symmetric objects $\left(M_{1}, \mu_{1}\right)$ and $\left(M_{2}, \mu_{2}\right)$ such that

$$
(E, \sigma) \perp\left(M_{1}, \mu_{1}\right) \simeq(F, \tau) \perp\left(M_{2}, \mu_{2}\right) .
$$

Definition 1.3. - If $\mathbf{C}$ is a small triangulated category with a duality $\left(^{*}, \delta, \varpi\right)$ and with $\frac{1}{2} \in \mathbf{C}$, then the $n$th derived Witt group of $\mathbf{C}$ is the set of Witt-equivalence classes of symmetric 
objects of $\mathbf{C}$ with respect to the $n$th shifted duality $\left(D_{n}, \delta_{n}, \varpi_{n}\right)$. It is written as $W^{n}(\mathbf{C})$ or $W^{n}\left(\mathbf{C},{ }^{*}, \delta, \varpi\right)$. The class of $(E, \sigma)$ in $W^{n}(\mathbf{C})$ is written $[E, \sigma]$. Addition is induced by the orthogonal direct sum $\perp$, while $-[E, \sigma]=[E,-\sigma]$.

The derived Witt groups satisfy $W^{n}(\mathbf{C})=W^{n+4}(\mathbf{C})$, while $W^{n+2}(\mathbf{C})=W^{n}\left(\mathbf{C},{ }^{*}, \delta,-\varpi\right)$ is the derived Witt group of skew-symmetric objects of $\mathbf{C}$ for the $n$th shifted duality.

A duality on an exact category $\mathbf{E}$ is a pair $\left({ }^{*}, \varpi\right)$ with ${ }^{*}: \mathbf{E}^{\mathrm{op}} \rightarrow \mathbf{E}$ a contravariant exact functor and $\varpi: 1 \rightarrow^{* *}$ an isomorphism of functors such that $\varpi_{E^{*}}: E^{*} \rightarrow E^{* * *}$ and $\varpi_{E}^{*}: E^{* * *} \rightarrow E^{*}$ are inverse isomorphisms for any object $E$. Transposes and symmetric objects in $\mathbf{E}$ are defined as in a triangulated category. A symmetric object $(E, \phi)$ is metabolic if there exists an exact sequence of the form $0 \rightarrow L \stackrel{\alpha}{\longrightarrow} E \stackrel{\alpha^{*} \phi}{\longrightarrow} L^{*} \rightarrow 0$. Then $L$ is a Lagrangian of $(E, \alpha)$. Witt equivalence and Witt groups are now defined as in (10) and Definition 1.3. The Witt groups of symmetric and of skew-symmetric objects of $\mathbf{E}$ are written $W(\mathbf{E})$ and $W^{-}(\mathbf{E})$, respectively.

A duality $\left({ }^{*}, \varpi\right)$ on an exact category $\mathbf{E}$ induces a triangulated duality $\left({ }^{*}, 1, \varpi\right)$ on the derived category $\mathbf{D}^{\mathrm{b}}(\mathbf{E})$ in a natural way (without fancy signs). There is the following result.

THEOREM 1.4 (Balmer [3, Theorem 4.3]). - Let $\mathbf{E}$ be a small exact category with duality and with $\frac{1}{2} \in \mathbf{E}$. Then for any $n$ there exist natural isomorphisms $W(\mathbf{E})=W^{4 n}\left(\mathbf{D}^{\mathrm{b}}(\mathbf{E})\right)$ and $W^{-}(\mathbf{E})=W^{4 n+2}\left(\mathbf{D}^{\mathrm{b}}(\mathbf{E})\right)$.

We will show in another paper that $W^{4 n+1}\left(\mathbf{D}^{\mathrm{b}}(\mathbf{E})\right)$ and $W^{4 n+3}\left(\mathbf{D}^{\mathrm{b}}(\mathbf{E})\right)$ are naturally isomorphic to Witt groups of skew-symmetric and symmetric formations in $\mathbf{E}$, respectively, as defined in Pardon [18] and similar to those of Ranicki [23,22].

Theorem 1.4 was proven in [3] for semisaturated exact categories. (These are the same as Gabriel-Roiter exact categories, cf. Keller [12].) However, Theorem 1.4 actually holds for all (Quillen) exact categories with duality. This is because any exact category has a semisaturation $\mathbf{E}^{\mathrm{sS}}$ satisfying a universal property that allows one to extend an exact duality from $\mathbf{E}$ to $\mathbf{E}^{\mathrm{ss}}$. The derived categories $\mathbf{D}^{\mathrm{b}}(\mathbf{E})$ and $\mathbf{D}^{\mathrm{b}}\left(\mathbf{E}^{\mathrm{ss}}\right)$ are equivalent (Neeman [15, Remark 1.12.3]), while the Witt groups $W(\mathbf{E})$ and $W\left(\mathbf{E}^{\mathrm{ss}}\right)$ are isomorphic. So the general case of Theorem 1.4 reduces to the semisaturated case. We omit the details. All the exact categories of sheaves and modules which appear in this paper are semisaturated.

\section{The long exact sequence}

We now review the localization long exact sequence for Witt groups in the triangulated version given in Balmer [2]. In particular we will prove this long exact sequence exists under slightly more general hypotheses than those given in [2]. We begin by recalling some definitions.

A saturated full triangulated subcategory $\mathbf{D}$ of a triangulated category $\mathbf{C}$ is a full additive subcategory which is closed under translations, mapping cones, direct summands, and isomorphisms. The quotient triangulated category $\mathbf{C} / \mathbf{D}$ of such a subcategory is obtained from $\mathbf{C}$ by using a calculus of fractions to invert the class $S$ of maps in $\mathbf{C}$ with cone in $\mathbf{D}$ (Verdier [26, § III.2]). The following result holds for triangulated categories satisfying a refined version (TR4+) of the octahedral axiom. For a description of this axiom, see [2, 11$]$ or [5, Remarque 1.1.13].

THEOREM 2.1 (Balmer [2, Theorem 6.2]). - Let $\mathbf{C}$ be a small triangulated category with duality satisfying (TR4+) and with $\frac{1}{2} \in \mathbf{C}$. Let $\mathbf{D} \subset \mathbf{C}$ be a saturated full triangulated subcategory stable under the duality. Then there is a long exact sequence of derived Witt groups

$$
\cdots \rightarrow W^{n}(\mathbf{D}) \rightarrow W^{n}(\mathbf{C}) \rightarrow W^{n}(\mathbf{C} / \mathbf{D}) \stackrel{\partial}{\longrightarrow} W^{n+1}(\mathbf{D}) \rightarrow \cdots
$$


The coboundary map $\partial$ is defined as follows. Any symmetric object in $\mathbf{C} / \mathbf{D}$ is isometric to a symmetric object of the form $(A, \bar{f})$ with $\bar{f}$ the image in $\mathbf{C} / \mathbf{D}$ of a symmetric morphism $f: A \rightarrow A^{\#}$ in $\mathbf{C}$. Since $\bar{f}$ is assumed to be an isomorphism in $\mathbf{C} / \mathbf{D}$, the mapping cone of $f$ lies in D. One sets $\partial[A, \bar{f}]:=[\operatorname{Cone}(A, f)]$.

The pair $(A, f)$ could be called a C-lattice of $(A, \bar{f})$.

If $\mathbf{E}$ is an exact category, then the homotopy category $\mathbf{K}(\mathbf{E})$ and all its subquotients satisfy (TR4+). This includes $\mathbf{D}^{\mathrm{b}}(\mathbf{E})$ and its subquotients and therefore all triangulated categories used in this paper.

Proof of Theorem 2.1. - This theorem was proven in Balmer [2, Theorem 6.2] under the hypothesis that $\mathbf{C} / \mathbf{D}$ is weakly cancellative. We show here how to remove this assumption. Actually, the only place where this condition is used in [2] is to prove exactness at $W^{n}(\mathbf{C})$ :

$$
W^{n}(\mathbf{D}) \rightarrow W^{n}(\mathbf{C}) \rightarrow W^{n}(\mathbf{C} / \mathbf{D})
$$

We can suppose $n=0$, replacing the duality by its shifted dualities to get the result for all $n$. Most of the proof of [2] extends here, and we will only present a slight modification of the final portion.

Let $S$ be the collection of those morphisms of $\mathbf{C}$ whose cone is in $\mathbf{D}$ or equivalently those morphisms in $\mathbf{C}$ which become isomorphisms in $\mathbf{C} / \mathbf{D}$. Then $\mathbf{C} / \mathbf{D}=S^{-1} \mathbf{C}$.

Let $(P, \varphi)$ be a symmetric object representing an element of the kernel:

$$
[P, \varphi] \in \operatorname{ker}(W(\mathbf{C}) \rightarrow W(\mathbf{C} / \mathbf{D}))
$$

By [2] Lemma 6.4, there exists a commutative diagram with exact rows

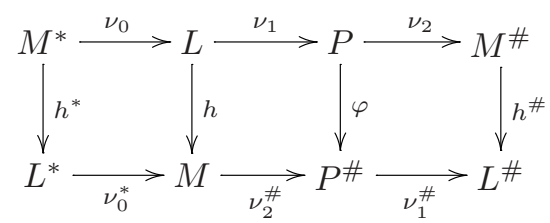

where $^{*}=T^{-1} \circ$ \# and where $h: L \rightarrow M=L^{\perp}$ belongs to $S$.

This $\left(L, \nu_{1}\right)$ is by definition a sublagrangian of $(P, \varphi)$. By [2] Lemma 4.13 applied to $\eta_{0}=h$, we get the existence of a morphism $\lambda: L \rightarrow L^{*}$ such that (A) $\nu_{0}^{*} \lambda \nu_{0}=0$, and (B) $\mu_{0}:=h+\nu_{0}^{*} \lambda$ is very good, which mainly means that we can construct a symmetric form on its cone which is Witt-equivalent to $(P, \varphi)$.

We claim that $\mu_{0}=h+\nu_{0}^{*} \lambda$ is in $S$, which implies that its cone is in $\mathbf{D}$.

To see this, we localize everything in $\mathbf{C} / \mathbf{D}=S^{-1} \mathbf{C}$, which means that $h$, which was in $S$, becomes an isomorphism. We prove that $\mu_{0}$ also localizes into an isomorphism. In $\mathbf{C} / \mathbf{D}$ define $x: L \rightarrow L$ by $x=h^{-1} \nu_{0}^{*} \lambda$. Applying $h^{-1}$ on the left to the localization of (A), we have $x \nu_{0}=0$ so that there exists $\bar{x}: P \rightarrow L$ with $x=\bar{x} \nu_{1}$. On the other hand, we have by (12) that $\nu_{1} x=\left(\varphi^{-1} \nu_{2}^{\#} h\right) x=\varphi^{-1} \nu_{2}^{\#} \nu_{0}^{*} \lambda$ by the very definition of $x$, and this implies that $\nu_{1} x=0$ since $\nu_{2}^{\#} \nu_{0}^{*}=\left(T \nu_{0} \nu_{2}\right)^{\#}=0$.

Therefore $x^{2}=\bar{x} \nu_{1} x=0$, and thus the endomorphism $1+x$ is an isomorphism. Finally, (B) gives directly $\mu_{0}=h(1+x)$, and so $\mu_{0}$ is an isomorphism in $\mathbf{C} / \mathbf{D}$, which was the claim.

Now it suffices to apply [2, Theorem 4.20] to the very good morphism $\mu_{0}$ to have a symmetric form $\psi$ on $R:=$ cone $\left(\mu_{0}\right) \in \mathbf{D}$ making it a symmetric object Witt-equivalent to $(P, \varphi)$. This 
implies that

$$
[P, \varphi]=[R, \psi] \in \operatorname{Im}(W(\mathbf{D}) \rightarrow W(\mathbf{C}))
$$

\section{The spectral sequence in a categorical context}

We now show that the long exact sequence of derived Witt groups of Theorem 2.1 generalizes to a spectral sequence when one has a triangulated category with duality with a finite filtration by saturated full triangulated subcategories invariant under the duality.

THEOREM 3.1. - Let $\mathbf{C}$ be a small triangulated category with duality satisfying (TR4+) and with $\frac{1}{2} \in \mathbf{C}$ and with a finite filtration by saturated full triangulated subcategories invariant under the duality

$$
\mathbf{C}=\mathbf{D}^{0} \supset \mathbf{D}^{1} \supset \cdots \supset \mathbf{D}^{e} \supset \mathbf{D}^{e+1} \simeq \mathbf{0} .
$$

Then there exists a spectral sequence of derived Witt groups converging to $E^{n}=W^{n}(\mathbf{C})$ with terms

$$
E_{1}^{p q}= \begin{cases}W^{p+q}\left(\mathbf{D}^{p} / \mathbf{D}^{p+1}\right) & \text { if } 0 \leqslant p \leqslant e \\ 0 & \text { otherwise. }\end{cases}
$$

Proof. - The filtration of $\mathbf{C}$ can be extended formally by setting $\mathbf{D}^{p}:=\mathbf{C}$ for $p<0$, and $\mathbf{D}^{p}:=\mathbf{D}^{e+1} \simeq \mathbf{0}$ for $p>e$. We now construct a spectral sequence with $E_{1}^{p q}:=W^{p+q}\left(\mathbf{D}^{p} / \mathbf{D}^{p+1}\right)$ for all $p, q$ which converges to $E^{n}=W^{n}(\mathbf{C})$.

For any $p, q$, let $A^{p q}:=W^{p+q}\left(\mathbf{D}^{p}\right)$, and let $E^{p q}:=W^{p+q}\left(\mathbf{D}^{p} / \mathbf{D}^{p+1}\right)$. By Theorem 2.1, the derived Witt groups of $\mathbf{D}^{p+1}, \mathbf{D}^{p}$, and $\mathbf{D}^{p} / \mathbf{D}^{p+1}$ fit into a long exact sequence

$$
\cdots \rightarrow A^{p+1, q-1} \rightarrow A^{p q} \rightarrow E^{p q} \stackrel{\partial}{\longrightarrow} A^{p+1, q} \rightarrow \cdots .
$$

If $A:=\bigoplus_{p, q} A^{p q}$ and $E:=\bigoplus_{p, q} E^{p q}$, then we deduce an exact sequence

$$
\cdots \rightarrow A \stackrel{i}{\longrightarrow} A \stackrel{j}{\longrightarrow} E \stackrel{k}{\longrightarrow} A \stackrel{i}{\longrightarrow} A \rightarrow \cdots
$$

with $i$ of bidegree $(-1,1)$, with $j$ of bidegree $(0,0)$ and with $k$ of bidegree $(1,0)$. Then (14) is an exact couple (cf. Weibel [28, §5.9]), and it determines a spectral sequence which starts with $E_{1}^{p q}=E^{p q}=W^{p+q}\left(\mathbf{D}^{p} / \mathbf{D}^{p+1}\right)$ and which abuts to

$$
E^{n}:=\lim _{\longrightarrow} A^{-p, n+p}=\underline{\lim } W^{n}\left(\mathbf{D}^{-p}\right)=W^{n}(\mathbf{C}) .
$$

The exact couple is bounded below because $A^{p q}=0$ for $p>e$. So the spectral sequence is convergent.

It is clear from the proof of the theorem that the spectral sequence exists even for an infinite filtration indexed by $p \in \mathbb{Z}$, and it converges to $W^{n}(\mathbf{C})$ if $\bigcup_{q \in \mathbb{Z}} \mathbf{D}^{q}=\mathbf{C}$ and $\mathbf{D}^{p} \simeq \mathbf{0}$ for $p \gg 0$.

Theorem 3.1 can also be proven by applying the construction of Cartan-Eilenberg [6] Chap. XV $\S 7$ to the groups $H(p, q)=\bigoplus_{n} W^{n}\left(\mathbf{D}^{p} / \mathbf{D}^{q}\right)$.

\section{Functoriality lemmas}

In the next two sections we prove some technical (and unsurprising) lemmas about Witt groups of triangulated categories which we will need when we discuss Witt groups of finite-length 
modules. We begin with some lemmas about the functoriality of derived Witt groups. We use the following definitions.

A duality-preserving functor from $(\mathbf{C}, D, \delta, \varpi)$ to $\left(\mathbf{C}^{\prime}, D^{\prime}, \delta^{\prime}, \varpi^{\prime}\right)$ is a pair $(F, \eta)$ with $F: \mathbf{C} \rightarrow \mathbf{C}^{\prime}$ an exact functor and with $\eta: F D \rightarrow D^{\prime} F$ an isomorphism of exact functors such that $T^{\prime} \eta= \pm \eta T^{-1}$ and such that the left-hand square
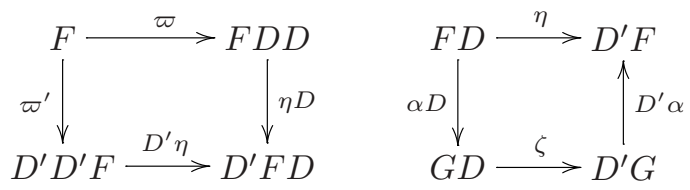

commutes. An isomorphism of duality-preserving functors $\alpha:(F, \eta) \rightarrow(G, \zeta)$ is an isomorphism of exact functors $\alpha: F \rightarrow G$ such that $\alpha T= \pm T^{\prime} \alpha$ and such that the right-hand square commutes. The sign in $T^{\prime} \eta= \pm \eta T^{-1}$ (resp. in $\alpha T= \pm T^{\prime} \alpha$ ) is + if $D$ and $D^{\prime}$ (resp. $F$ and $G$ ) are of the same parity, while it is - if they are of opposite parity.

Part (a) of the next lemma is a generalization of Balmer [1, $\S 1.19]$.

LEMMA 4.1.- (a) A duality-preserving functor induces morphisms of derived Witt groups $W^{n}(\mathbf{C}, D, \delta, \varpi) \rightarrow W^{n}\left(\mathbf{C}^{\prime}, D^{\prime}, \delta^{\prime}, \varpi^{\prime}\right)$.

(b) Isomorphic duality-preserving functors induce the same morphisms.

(c) A duality-preserving equivalence induces isomorphisms of the derived Witt groups.

Proof. - (a) Given a map $\phi: A \rightarrow D_{n} B$ in C, let $\widetilde{F}(\phi)$ be the composite map

$$
F A \stackrel{F \phi}{\longrightarrow} F D_{n} B \stackrel{\eta}{\longrightarrow} D_{n}^{\prime} F B .
$$

Since $\eta$ commutes with $T^{n}$ up to sign, the left-hand square of (15) commutes for $D_{n}$ and $D_{n}^{\prime}$ as soon as it commutes for $D$ and $D^{\prime}$. One easily checks that this commutativity implies that $\widetilde{F}\left(\phi^{\mathrm{t}}\right)=\widetilde{F}(\phi)^{\mathrm{t}}$ and that $\widetilde{F}$ preserves Lagrangian diagrams of the form of (9). In addition $\widetilde{F}$ preserves isomorphisms because $\eta$ is an isomorphism. So the map $W^{n}(\mathbf{C}) \rightarrow W^{n}\left(\mathbf{C}^{\prime}\right)$ sending $[B, \phi] \mapsto[F(B), \widetilde{F}(\phi)]$ is well-defined.

(b) If $\alpha:(F, \eta) \rightarrow(G, \zeta)$ is an isomorphism of duality-preserving functors, and if $(B, \phi)$ is a symmetric object in $\mathbf{C}$, then the commutativity of the right-hand square of (15) implies that $(F(B), \widetilde{F}(\phi))$ and $(G(B), \widetilde{G}(\phi))$ are isometric in $\mathbf{C}^{\prime}$. So $[F(B), \widetilde{F}(\phi)]=[G(B), \widetilde{G}(\phi)]$.

(c) If $F$ is fully faithful and essentially surjective, and if $\phi: A \rightarrow D_{n}^{\prime} A$ is a symmetric isomorphism in $\mathbf{C}^{\prime}$, then there exists a $B$ in $\mathbf{C}$ and an isomorphism $f: F(B) \rightarrow A$. Consequently $(A, \phi)$ is isometric to $\left(F(B),\left(D_{n}^{\prime} f\right) \phi f\right)$. But since $F$ is fully faithful, there exists an isomorphism $g: B \rightarrow D_{n} B$ such that $\widetilde{F}(g)=\left(D_{n}^{\prime} f\right) \phi f$. So the map on derived Witt groups is surjective. A similar argument shows that the map is injective.

We leave the proof of the next lemma to the reader.

LEMMA 4.2. - The spectral sequence for Witt groups of filtered triangulated categories with duality of Theorem 3.1 is functorial for duality-preserving functors which are compatible with the filtration.

The statements in the next lemma apply both to exact and to triangulated categories. Despite the obvious-sounding statements, complete proofs require diagram chases to verify all the technical details.

LEMma 4.3. - (a) A contravariant exact functor isomorphic to a duality can be made into a duality. 
(b) An exact functor isomorphic to a duality-preserving functor can be made dualitypreserving.

(c) A category equivalent to a category with duality can be equipped with a duality so that the equivalence is duality-preserving.

(d) An equivalence quasi-inverse to a duality-preserving equivalence can be made dualitypreserving.

Sketch of proof. - Parts (a) and (b) are straightforward and are left to the reader. Parts (c) and (d) require the use of adjoint equivalences. We sketch the proof of (d).

(d) Let $(F, \eta):(\mathbf{C}, D, \delta, \varpi) \rightarrow\left(\mathbf{C}^{\prime}, D^{\prime}, \delta^{\prime}, \varpi^{\prime}\right)$ be a duality-preserving equivalence, and let $G: \mathbf{C}^{\prime} \rightarrow \mathbf{C}$ be a quasi-inverse of $F$. We need to find an isomorphism of functors $\gamma: G D^{\prime} \rightarrow D G$ so that $(G, \gamma)$ is duality-preserving.

By Mac Lane [13, IV.1], $F$ and $G$ can be completed to an adjunction of equivalences, i.e. there exist isomorphisms of functors $\varepsilon: 1_{\mathbf{C}} \rightarrow G F$ and $\zeta: 1_{\mathbf{C}^{\prime}} \rightarrow F G$ such that in each pair of isomorphisms

$$
G \underset{G \zeta}{\stackrel{\varepsilon G}{\longrightarrow}} G F G \quad F \underset{\zeta F}{\stackrel{F \varepsilon}{\longrightarrow}} F G F
$$

the two arrows coincide. Moreover, $\varepsilon$ and $\zeta$ commute with the translation up to sign in the triangulated case.

Now let $\gamma^{-1}$ be the composite isomorphism

$$
D G \stackrel{\varepsilon D G}{\longrightarrow} G F D G \stackrel{G \eta G}{\longrightarrow} G D^{\prime} F G \stackrel{G D^{\prime} \zeta}{\longrightarrow} G D^{\prime} .
$$

A long diagram chase (which we omit) shows that if $\eta$ makes the left-hand square of (15) commute, then the left-hand square of
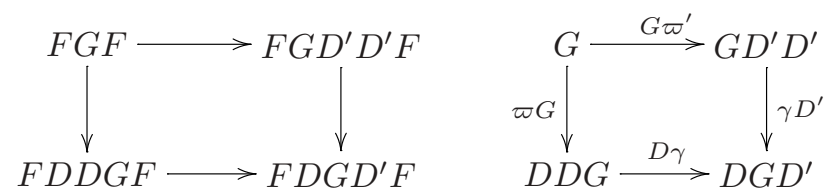

commutes. Since $F$ is an equivalence, this implies that the right-hand square also commutes, i.e. $(G, \gamma)$ is duality-preserving.

\section{Witt groups of abelian categories}

We now discuss derived Witt groups of abelian categories with an exact duality. With our definitions an exact duality is left and right exact and is reflexive on all objects, so many functors commonly called 'duality' do not fit the bill. For even Witt groups, there is the following result.

Proposition 5.1 (Quebbemann-Scharlau-Schulte [21]).- Let A be a small abelian category with an exact duality in which all objects are of finite length, and let $\mathbf{A}^{0} \subset \mathbf{A}$ be the full subcategory of semisimple objects. Then the inclusion induces isomorphisms of Witt groups $W\left(\mathbf{A}^{0}\right)=W(\mathbf{A})$ and $W^{-}\left(\mathbf{A}^{0}\right)=W^{-}(\mathbf{A})$.

Proposition 5.1 is a consequence of the symmetric Jordan-Hölder Theorem. It is proven in [21] Corollary 6.9 subject to a condition which is verified in [21] Theorem 6.10 when all objects of $\mathbf{A}$ are of finite length. 
The result concerning the odd Witt groups of an abelian category requires some basic facts about canonical truncations. For any integer $n$ let $\mathbf{D}^{\mathrm{b}, \leqslant n}(\mathbf{A})$ and $\mathbf{D}^{\mathrm{b}, \geqslant n}(\mathbf{A})$ be the full additive subcategories of $\mathbf{D}^{\mathrm{b}}(\mathbf{A})$ such that

$$
\begin{aligned}
& \mathrm{ob} \mathbf{D}^{\mathrm{b}, \leqslant n}(\mathbf{A}):=\left\{X \mid H^{i}(X)=0 \text { for all } i>n\right\}, \\
& \mathrm{ob} \mathbf{D}^{\mathrm{b}, \geqslant n}(\mathbf{A}):=\left\{X \mid H^{i}(X)=0 \text { for all } i<n\right\} .
\end{aligned}
$$

Then for any complex $Y$ in $\mathbf{D}^{\mathrm{b}}(\mathbf{A})$ and any $n$ there exists an exact triangle

$$
T^{-1}\left(\tau^{\geqslant n+1} Y\right) \rightarrow \tau^{\leqslant n} Y \rightarrow Y \rightarrow \tau^{\geqslant n+1} Y
$$

with $\tau^{\leqslant n} Y$ in $\mathbf{D}^{\mathrm{b}, \leqslant n}(\mathbf{A})$ and with $\tau^{\geqslant n+1} Y$ in $\mathbf{D}^{\mathrm{b}, \geqslant n+1}(\mathbf{A})$. This triangle is unique up to unique isomorphism (Beilinson-Bernstein-Deligne [5], Proposition 1.3.3).

Proposition 5.2. - Let $\mathbf{A}$ be a small abelian category with an exact duality and with $\frac{1}{2} \in \mathbf{A}$. Then its odd derived Witt groups are $W^{2 n+1}\left(\mathbf{D}^{\mathrm{b}}(\mathbf{A})\right)=0$.

Proof. - Let $C \mapsto C^{\#}:=T^{2 n+1}\left(C^{*}\right)$ denote the shifted duality on $\mathbf{D}^{\mathrm{b}}(\mathbf{A})$, and suppose that $(C, \phi)$ is a complex which is symmetric with respect to this shifted duality. We will show that the $\tau^{\leqslant-n-1} C$ of (16) is a Lagrangian of $(C, \phi)$, so $[C, \phi]=0$. The details are as follows.

Using the truncation exact triangle (16) and applying the (-1)-exact duality gives a diagram of solid arrows whose rows are exact triangles:

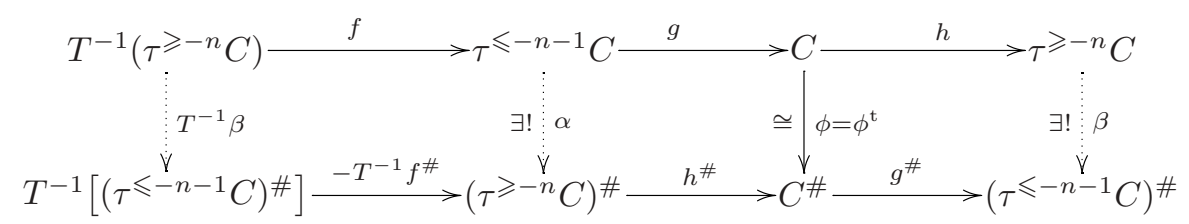

Since the duality on $\mathbf{A}$ is exact, the derived duality acts on the cohomology objects as $H^{i}\left(X^{\#}\right) \cong\left[H^{-2 n-1-i}(X)\right]^{*}$, and so it exchanges $\mathbf{D}^{\mathrm{b}, \leqslant-n-1}(\mathbf{A})$ and $\mathbf{D}^{\mathrm{b}, \geqslant-n}(\mathbf{A})$. As a result, we see that $\tau^{\leqslant-n-1} C$ and $\left(\tau^{\geqslant-n} C\right)^{\#}$ are in the first subcategory, while $\tau^{\geqslant-n} C$ and $\left(\tau^{\leqslant-n-1} C\right)^{\#}$ are in the second subcategory. Since a truncation exact triangle (16) is unique up to unique isomorphism, there exist unique isomorphisms $\alpha$ and $\beta$ making the diagram above commute. Moreover, since $\phi=\phi^{\mathrm{t}}$, the diagram also commutes if we replace $\alpha, \beta$ by $\beta^{\mathrm{t}}$, $\alpha^{\mathrm{t}}$. Since $\alpha$ and $\beta$ are unique, this gives $\beta=\alpha^{\mathrm{t}}$. Taking into account that $\alpha^{\mathrm{t}}=\alpha^{\#} \varpi_{2 n+1}$ by definition, that $\varpi_{2 n}=-\varpi_{2 n+1}$ by (8), and that $T^{-1} \varpi_{2 n}=\varpi_{2 n}$ by Definition 1.1, we get a commutative diagram with exact rows

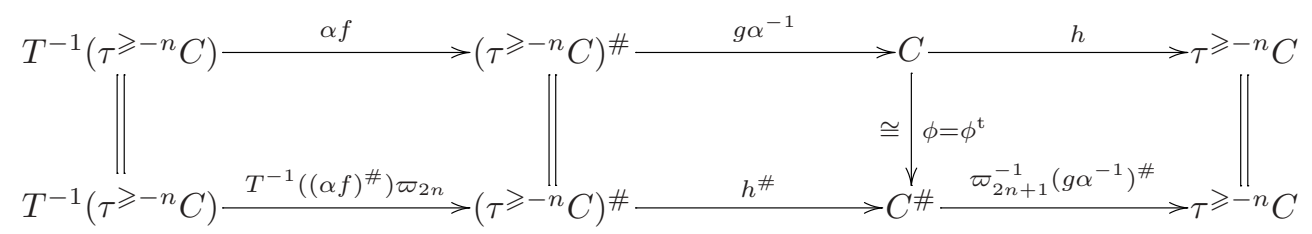

The last map on the bottom row is the transpose of $g \alpha^{-1}$, while the first map on the bottom row is the transpose of $\alpha f$ with respect to the $2 n$th shifted duality. Therefore our diagram is of the form of diagram (9). So $[C, \phi]=[\operatorname{Cone}(A, u)]=0$ in $W^{2 n+1}(\mathbf{A})$. 


\section{Witt groups of finite-length modules}

Let $\mathcal{O}$ be a regular local ring, and let $\mathbf{D}_{f}^{\mathrm{b}}\left(\mathrm{VB}_{\mathcal{O}}\right)$ be the derived category of bounded complexes of free $\mathcal{O}$-modules with finite length homology, equipped with the 'usual' duality defined below. This section will be devoted to proving the following result:

THEOREM 6.1 (Dévissage). - Let $\mathcal{O}$ be a regular local ring with residue field $k$ such that $\operatorname{char}(k) \neq 2$. Then $W^{n}\left(\mathbf{D}_{\mathrm{fl}}^{\mathrm{b}}\left(\mathrm{VB}_{\mathcal{O}}\right)\right) \cong W(k)$ if $n \equiv \operatorname{dim} \mathcal{O}(\bmod 4)$, and $W^{n}\left(\mathbf{D}_{\mathrm{fl}}^{\mathrm{b}}\left(\mathrm{VB}_{\mathcal{O}}\right)\right)=0$ otherwise.

In the course of proving the theorem, we will also prove the following known result. Let $\mathcal{O}$-fl-mod be the abelian category of $\mathcal{O}$-modules of finite length. It has a 'standard' duality which we also define below (19).

PROPOSITION 6.2. - Let $\mathcal{O}$ be a regular local ring with residue field $k$ such that $\operatorname{char}(k) \neq 2$. Then $W(\mathcal{O}$-fl-mod $) \cong W(k)$, while $W^{-}(\mathcal{O}$-fl-mod $)=0$.

The 'usual' duality on $\operatorname{VB}_{\mathcal{O}}$ is $\left({ }^{*}, \varpi\right)$ where $E^{*}=\operatorname{Hom}_{\mathcal{O}}(E, \mathcal{O})$, and where $\varpi_{E}: E \rightarrow E^{* *}$ is the evaluation map. This duality extends to $\mathbf{D}_{\mathrm{f}}^{\mathrm{b}}\left(\mathrm{VB}_{\mathcal{O}}\right)$.

The 'standard' duality on $\mathcal{O}$-fl-mod is defined as follows. Let $e:=\operatorname{dim} \mathcal{O}$, and let $M^{\vee}:=\operatorname{Ext}_{\mathcal{O}}^{e}(M, \mathcal{O})$. Define $\widetilde{\varpi}_{M}: M \rightarrow M^{\vee \vee}$ using the commutative diagram between projective resolutions

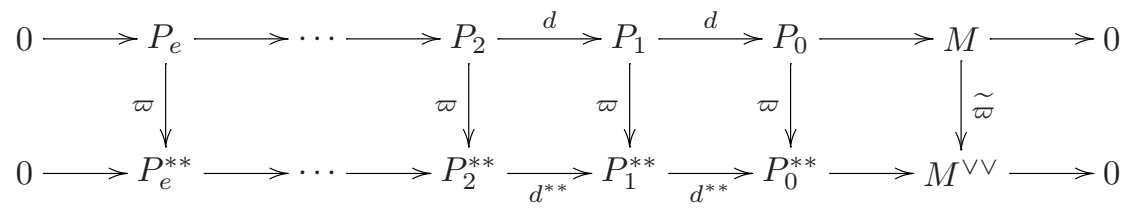

The 'standard' exact duality on $\mathcal{O}$-fl-mod is the duality with sign

$$
\left({ }^{\vee},(-1)^{e(e-1) / 2} \widetilde{\varpi}\right) .
$$

Now let $k$-mod be the category of finite-dimensional vector spaces over $k$, equipped with the duality given by $V^{\prime}:=\operatorname{Hom}_{k}(V, k)$ and the evaluation maps ev: $V \rightarrow V^{\prime \prime}$. Let $\mathcal{O}$-fl-mod ${ }^{0} \subset \mathcal{O}$-fl-mod be the full subcategory of semisimple objects. The main step in the proof of Proposition 6.2 is the following lemma.

LEMMA 6.3. - There is an equivalence of semisimple abelian categories with duality between $\left(\mathcal{O}\right.$-fl-mod $\left.{ }^{0},{ }^{\vee},(-1)^{e(e-1) / 2} \widetilde{\varpi}\right)$ and $\left(k\right.$-mod, ${ }^{\prime}$, ev $)$.

Proof. - In order to make the various dualities and equivalences more concrete and less liable to try to wriggle away when we try to work with them, we will replace the natural isomorphism of categories $k$-mod $\cong \mathcal{O}$-fl-mod ${ }^{0}$ with a diagram of equivalences

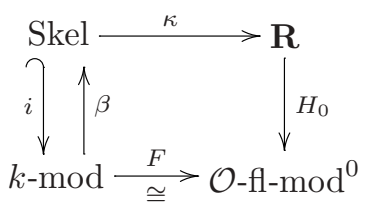

which we will define in a moment. However, we will have $F i=H_{0} \kappa$. So once we show that $i, \kappa$, and $H_{0}$ are duality-preserving, then the quasi-inverse $\beta$ of $i$ is also duality-preserving 
by Lemma 4.3(d). So $F$, being isomorphic to $H_{0} \kappa \beta$, will also be duality-preserving by Lemma 4.3(b).

Let Skel $\subset k$-mod be the full subcategory whose objects are the vector spaces $k^{n}$ with $n \geqslant 0$ and whose morphisms are matrices. The functor ' on Skel fixes the objects and transposes the matrices. The restriction of ev to Skel is the identity. One checks that the inclusion $i$ is dualitypreserving. Its quasi-inverse $\beta: k$-mod $\rightarrow$ Skel amounts to choosing a basis for every vector space.

Let $\mathbf{R} \subset \mathbf{K}^{\mathrm{b}}\left(\mathrm{VB}_{\mathcal{O}}\right)=\mathbf{D}^{\mathrm{b}}\left(\mathrm{VB}_{\mathcal{O}}\right)$ be the full subcategory of complexes

$$
0 \rightarrow R_{e} \rightarrow \cdots \rightarrow R_{2} \stackrel{d}{\longrightarrow} R_{1} \stackrel{d}{\longrightarrow} R_{0} \rightarrow 0
$$

of finitely generated free $\mathcal{O}$-modules such that $H_{i}(R)=0$ for $i \neq 0$ and such that $\mathfrak{m} \cdot H_{0}(R)=0$ for $\mathfrak{m} \subset \mathcal{O}$ the maximal ideal. Then $R^{\vee}$ is the complex

$$
0 \rightarrow R_{0}^{*} \rightarrow \cdots \rightarrow R_{e-2}^{*} \stackrel{d^{*}}{\longrightarrow} R_{e-1}^{*} \stackrel{d^{*}}{\longrightarrow} R_{e}^{*} \rightarrow 0
$$

while $\widetilde{\varpi}: R \rightarrow R^{\vee \vee}$ is as in (18). The functor $H_{0}$ is clearly duality-preserving from $\left(\mathbf{R},{ }^{\vee},(-1)^{e(e-1) / 2} \widetilde{\varpi}\right)$ to $\left(\mathcal{O}\right.$-fl-mod $\left.{ }^{0}, \vee,(-1)^{e(e-1) / 2} \widetilde{\varpi}\right)$.

Let $K$ be a minimal free resolution of the $\mathcal{O}$-module $k$. Let $\kappa:$ Skel $\rightarrow \mathbf{R}$ be the functor which sends $k^{n} \mapsto K^{\oplus n}$ and which sends an arrow $\alpha: k^{n} \rightarrow k^{m}$ to the homotopy class of maps between the minimal free resolutions $K^{\oplus n} \rightarrow K^{\oplus m}$ which lift $\alpha$. To check that $\kappa$ is duality-preserving, we need an isomorphism of functors $\eta: \kappa \circ^{\prime} \rightarrow^{\vee} \circ \kappa$ such that the left-hand square of (15) commutes. Since $\kappa$ is compatible with the direct sum, it is enough to give an isomorphism $\eta_{k}: K \rightarrow K^{\vee}$ such that

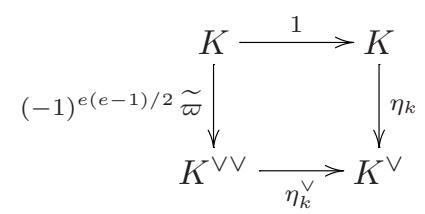

commutes, i.e. we want $\eta_{k}$ to be an isomorphism which is symmetric for the duality with sign $(-1)^{e(e-1) / 2}$.

We now describe $K$ explicitly. Let $F$ be a free $\mathcal{O}$-module of rank $e$ with basis $f_{1}, \ldots, f_{e}$, and let $x_{1}, \ldots, x_{e}$ be a regular sequence generating the maximal ideal $\mathfrak{m} \subset \mathcal{O}$. Let $K$ be the complex

$$
0 \rightarrow \mathcal{O} \rightarrow F \rightarrow \Lambda^{2} F \rightarrow \cdots \rightarrow \Lambda^{e-1} F \rightarrow \Lambda^{e} F \rightarrow 0
$$

with differential $d(m)=\sum_{i=1}^{e} x_{i} f_{i} \wedge m$. We will give the chain isomorphism $\eta_{k}: K \rightarrow K^{\vee}$ with components $K_{j} \rightarrow K_{e-j}^{*}$ by specifying the corresponding bilinear maps

$$
\varphi_{j}: K_{j} \times K_{e-j}=\left(\Lambda^{e-j} F\right) \times\left(\Lambda^{j} F\right) \rightarrow \mathcal{O} .
$$

We fix an isomorphism $\Lambda^{e} F \cong \mathcal{O}$ and set $\varphi_{j}(m, n)=\varepsilon_{j}(m \wedge n)$ with $\varepsilon_{j}=(-1)^{e j-j(j+1) / 2}$. The induced maps $K_{j} \rightarrow K_{e-j}^{*}$ are compatible with the differentials of the chain complexes because for all $j$ and all $m \in K_{j}$ and all $n \in K_{e-j+1}$ we have $\varphi_{j}(m, d n)=\varphi_{j-1}(d m, n)$ or equivalently

$$
\varepsilon_{j}\left(m \wedge \sum x_{i} f_{i} \wedge n\right)=\varepsilon_{j-1}\left(\sum x_{i} f_{i} \wedge m \wedge n\right)
$$


So the $K_{j} \rightarrow K_{e-j}^{*}$ are the components of a chain map $\eta_{k}: K \rightarrow K^{\vee}$. Moreover, the maps $K_{j} \rightarrow$ $K_{e-j}^{*}$ are isomorphisms because the exterior monomials in $K_{j}=\Lambda^{e-j} F$ and the complementary exterior monomials in $K_{e-j}=\Lambda^{j} F$ form module bases which are $\varphi_{j}$-dual up to sign. So $\eta_{k}: K \rightarrow K^{\vee}$ is a chain isomorphism. Finally, one checks that

$$
\varphi_{e-j}(n, m)=(-1)^{e(e-1) / 2} \varphi_{j}(m, n) .
$$

So $\eta_{k}: K \rightarrow K^{\vee}$ is symmetric for the duality with sign $(-1)^{e(e-1) / 2}$, i.e. the diagram (20) commutes. This completes the proof of the lemma.

Proof of Proposition 6.2. - Apply Proposition 5.1 and Lemma 6.3.

To complete the proof of Theorem 6.1 we require the following lemma.

LEMMA 6.4. - There is an equivalence of triangulated categories with duality between $\left(\mathbf{D}_{\mathrm{f}}^{\mathrm{b}}\left(\mathrm{VB}_{\mathcal{O}}\right), D_{e}, \delta_{e}, \varpi_{e}\right)$ and $\left(\mathbf{D}^{\mathrm{b}}(\mathcal{O}\right.$-fl-mod $\left.),{ }^{\vee}, 1,(-1)^{e(e-1) / 2} \widetilde{\varpi}\right)$.

Proof. - There are natural exact functors

$$
\mathbf{D}_{\mathrm{f}}^{\mathrm{b}}\left(\mathrm{VB}_{\mathcal{O}}\right) \rightarrow \mathbf{D}_{\mathrm{fl}}^{\mathrm{b}}(\mathcal{O} \text {-mod }) \leftarrow \mathbf{D}^{\mathrm{b}}(\mathcal{O} \text {-fl-mod })
$$

The first functor is an equivalence of triangulated categories because $\mathrm{VB}_{\mathcal{O}} \subset \mathcal{O}$-mod is the full subcategory of projectives, and $\mathcal{O}$-mod is of finite global projective dimension. The second functor is an equivalence of triangulated categories by Keller [11] § 1.15, Example (b).

Let $T_{\text {uns }}$ be the unsigned translation functor which translates a complex to the left one place without changing the sign of its differential. Let $D_{e}^{\prime}:=T_{\mathrm{uns}}^{e} 0^{*}$ be the unsigned shifted duality on $\mathbf{D}^{\mathrm{b}}\left(\mathrm{VB}_{\mathcal{O}}\right)$. It is 1-exact. We claim that there are natural equivalences of triangulated categories with duality

$$
\begin{aligned}
\left(\mathbf{D}^{\mathrm{b}}(\mathcal{O} \text {-fl-mod }),{ }^{\vee}, 1, \widetilde{\varpi}\right) & \simeq\left(\mathbf{D}_{\mathrm{fl}}^{\mathrm{b}}\left(\mathrm{VB}_{\mathcal{O}}\right), D_{e}^{\prime}, 1, \varpi\right), \\
\left(\mathbf{D}_{\mathrm{fl}}^{\mathrm{b}}\left(\mathrm{VB}_{\mathcal{O}}\right), D_{e}^{\prime}, 1, \varpi\right) & \simeq\left(\mathbf{D}_{\mathrm{fl}}^{\mathrm{b}}\left(\mathrm{VB}_{\mathcal{O}}\right), D_{e},(-1)^{e},(-1)^{e} \varpi\right) .
\end{aligned}
$$

Multiplying by $(-1)^{e(e-1) / 2}$ will then give the lemma because $\delta_{e}=(-1)^{e}$ and $\varpi_{e}=$ $(-1)^{e(e+1) / 2}$ by $(8)$.

The second equivalence (25) is given by the duality-preserving functor $\left(1_{\mathbf{D}_{\mathrm{fl}}^{\mathrm{b}}\left(\mathrm{VB}_{\mathcal{O}}\right)}, \eta\right)$ where $\eta: D_{e}^{\prime} \rightarrow D_{e}$ is the isomorphism of functors of the form

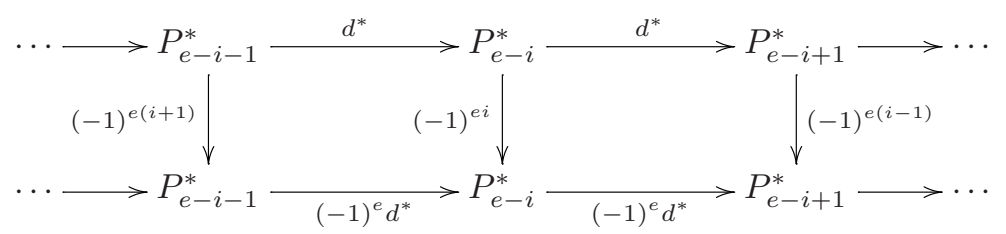

The first equivalence (24) will be obtained from a diagram of duality-preserving equivalences

$$
\mathbf{D}^{\mathrm{b}}(\mathcal{O} \text {-fl-mod }) \underset{f}{\stackrel{p}{\longrightarrow}} \mathbf{C} \stackrel{q}{\longrightarrow} \mathbf{D}_{\mathrm{fl}}^{\mathrm{b}}\left(\mathrm{VB}_{\mathcal{O}}\right)
$$

which we will define below. We will show that $p$ and $q$ are duality-preserving equivalences. The quasi-inverse $f$ of $p$ will then be a duality-preserving equivalence by Lemma 4.3(d), and $q f$ will be the equivalence of (24). 
We now specify the category $\mathbf{C}$. Its objects are bounded double complexes with commuting squares of the form

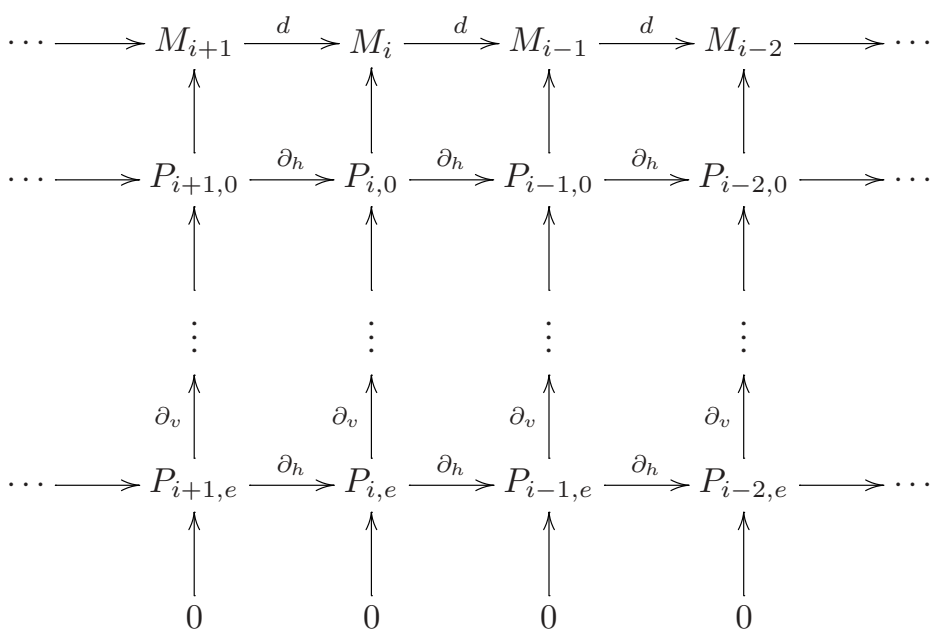

in which the $M_{i}$ are in $\mathcal{O}$-fl-mod, the $P_{i j}$ are in $\mathrm{VB}_{\mathcal{O}}$, and the $i$ th column is a free resolution of $M_{i}$. We denote such an object by $(P \rightarrow M)$. Write $\operatorname{Tot}(P)$ for the complex with terms $\operatorname{Tot}(P)_{n}=\bigoplus_{i+j=n} P_{i j}$ and with differential $\partial$ such that $\left.\partial\right|_{P_{i j}}=\left.\left(\partial_{h}+(-1)^{i} \partial_{v}\right)\right|_{P_{i j}}$. The group of morphisms from $(P \rightarrow M)$ to $\left(P^{\prime} \rightarrow M^{\prime}\right)$ is defined to be

$$
\operatorname{Hom}_{\mathbf{D}^{\mathrm{b}}(\mathcal{O}-\mathrm{fl}-\mathrm{mod})}\left(M, M^{\prime}\right)=\operatorname{Hom}_{\mathbf{D}^{\mathrm{b}}\left(\operatorname{VB}_{\mathcal{O}}\right)}\left(\operatorname{Tot}(P), \operatorname{Tot}\left(P^{\prime}\right)\right) .
$$

The two sides of the equation are the same because of the equivalence (23). The projection functors given by $p(P \rightarrow M)=M$ and $q(P \rightarrow M)=\operatorname{Tot}(P)$ are clearly equivalences.

The duality ${ }^{\vee}$ on $\mathbf{C}$ sends the double complex $(P \rightarrow M)$ above to

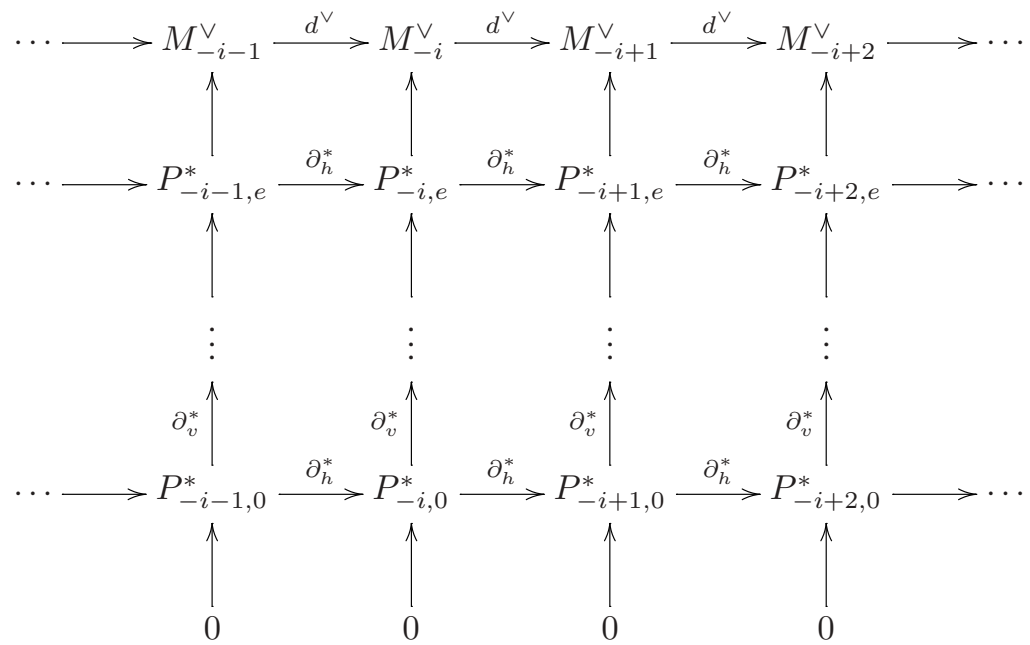

Then $p\left((P \rightarrow M)^{\vee}\right)=M^{\vee}$ and $q\left((P \rightarrow M)^{\vee}\right)=D_{e}^{\prime} \operatorname{Tot}(P)$. The isomorphism $1_{\mathbf{C}} \rightarrow \vee \vee$ is defined by sewing together $\widetilde{\varpi}: M \rightarrow M^{\vee \vee}$ and $\varpi: P \rightarrow P^{* *}$. The equivalences $p$ and $q$ clearly 
preserve the dualities. Thus the functors of the diagram (27) are duality-preserving equivalences, establishing the equivalence (24) and proving the lemma.

Proof of Theorem 6.1. - The duality-preserving equivalence of triangulated categories of Lemma 6.4 produces natural isomorphisms

$$
W^{n+e}\left(\mathbf{D}_{\mathrm{fl}}^{\mathrm{b}}\left(\mathrm{VB}_{\mathcal{O}}\right)\right)=W^{n}\left(\mathbf{D}^{\mathrm{b}}(\mathcal{O} \text {-fl-mod })\right)
$$

for every $n$. Since $\mathcal{O}$-fl-mod is abelian, Theorem 1.4 and Proposition 5.2 give

$$
W^{n}\left(\mathbf{D}^{\mathrm{b}}(\mathcal{O} \text {-fl-mod })\right)= \begin{cases}W(\mathcal{O} \text {-fl-mod }) & \text { if } n \equiv 0(\bmod 4), \\ W^{-}(\mathcal{O} \text {-fl-mod }) & \text { if } n \equiv 2(\bmod 4), \\ 0 & \text { if } n \equiv 1 \text { or } 3(\bmod 4)\end{cases}
$$

Apply Proposition 6.2 to complete the proof.

\section{The Gersten-Witt spectral sequence}

In this section we obtain our Gersten-Witt spectral sequence by applying the abstract construction of Theorem 3.1 to the derived category of bounded complexes of vector bundles on a regular separated noetherian scheme $X$. We also show that the spectral sequence is functorial for inclusions $U \subset X$ with $U$ an open subscheme or with $U=\operatorname{Spec}\left(\mathcal{O}_{X, x}\right)$.

Let $X$ be a regular separated noetherian scheme. Let $\mathrm{VB}_{X}$ be the exact category of locally free sheaves of finite rank on $X$, and let $\mathbf{D}^{\mathrm{b}}\left(\mathrm{VB}_{X}\right)$ be its bounded derived category. A line bundle $L$ on $X$ induces a duality $\left(D_{L}, \varpi_{L}\right)$ on $\operatorname{VB}_{X}$ where $D_{L} \mathcal{E}:=\mathcal{H}_{o} m_{\mathcal{O}_{X}}(\mathcal{E}, L)$, and where $\varpi_{L}$ is the evaluation map. This duality extends to a 'derived' duality on $\mathbf{D}^{\mathrm{b}}\left(\mathrm{VB}_{X}\right)$. If $\frac{1}{2} \in \Gamma\left(X, \mathcal{O}_{X}\right)$, then the $n$th derived Witt group of $X$ with respect to $L$ is

$$
W^{n}(X, L):=W^{n}\left(\mathbf{D}^{\mathrm{b}}\left(\mathrm{VB}_{X}\right), D_{L}, 1, \varpi_{L}\right) .
$$

If $\mathcal{E}^{*}$ is a bounded complex of vector bundles, then set

$$
\operatorname{Supp}\left(\mathcal{E}^{*}\right):=\bigcup_{i} \operatorname{Supp} H^{i}\left(\mathcal{E}^{*}\right)=\left\{x \in X \mid \mathcal{E}^{*} \text { is not exact at } x\right\} .
$$

For each integer $p \geqslant 0$ let $\mathbf{D}_{X}^{p} \subset \mathbf{D}^{\mathrm{b}}\left(\mathrm{VB}_{X}\right)$ be the full triangulated subcategory of complexes with support of codimension $\geqslant p$. If $\operatorname{dim} X=e$, then we have a filtration by saturated full triangulated subcategories

$$
\mathbf{D}^{\mathrm{b}}\left(\mathrm{VB}_{X}\right)=\mathbf{D}_{X}^{0} \supset \mathbf{D}_{X}^{1} \supset \cdots \supset \mathbf{D}_{X}^{e} \supset \mathbf{D}_{X}^{e+1} \simeq \mathbf{0} .
$$

Let $X^{(p)}$ be the set of points of codimension $p$ of $X$. The following result is well known.

Proposition 7.1.- If $X$ is a regular separated noetherian scheme, then there are equivalences $\mathbf{D}_{X}^{p} / \mathbf{D}_{X}^{p+1} \simeq \coprod_{x_{p} \in X^{(p)}} \mathbf{D}_{\mathrm{fl}}^{\mathrm{b}}\left(\mathrm{VB}_{\mathcal{O}_{x_{p}}}\right)$ of triangulated categories.

Proof. - Let $\mathbf{M}_{X}^{p}$ be the category of coherent $\mathcal{O}_{X}$-modules with support of codimension $\geqslant p$. It is easy to see that the functor sending a sheaf to its stalks gives an identification of the quotient abelian category:

$$
\mathbf{M}_{X}^{p} / \mathbf{M}_{X}^{p+1} \simeq \coprod_{x_{p} \in X^{(p)}} \mathcal{O}_{x_{p}} \text {-fl-mod. }
$$


The argument of Keller [11] 11.15 , Lemma and Example (b), applies to a separated noetherian $X$ and shows that the bounded derived category of the lefthand side is equivalent to $\mathbf{D}^{\mathrm{b}}\left(\mathbf{M}_{X}^{p}\right) / \mathbf{D}^{\mathrm{b}}\left(\mathbf{M}_{X}^{p+1}\right)$. The same criterion shows that $\mathbf{D}^{\mathrm{b}}\left(\mathbf{M}_{X}^{p}\right) \simeq \mathbf{D}_{\mathbf{M}_{X}^{p}}^{\mathrm{b}}\left(\mathrm{Coh}_{X}\right)$, and this is equivalent to $\mathbf{D}_{X}^{p}$ because $X$ is regular, separated, and noetherian. By Lemma 6.4 the bounded derived category of the righthand side of (30) is equivalent to $\mathbf{D}_{\mathrm{fl}}^{\mathrm{b}}\left(\mathrm{VB}_{\mathcal{O}_{x_{p}}}\right)$.

THEOREM 7.2. - Let $X$ be a regular separated noetherian scheme of finite Krull dimension such that $2 \in \Gamma\left(X, \mathcal{O}_{X}\right)$ is invertible, and let $L$ be a line bundle on $X$. Let $X^{(i)}$ be the set of points of codimension $i$ in $X$. Then there exists a spectral sequence with terms

$$
E_{1}^{p q} \cong \begin{cases}\bigoplus_{x_{p} \in X^{(p)}} W\left(k\left(x_{p}\right)\right), & \text { if } 0 \leqslant p \leqslant \operatorname{dim}(X), \text { and } q \equiv 0(\bmod 4) \\ 0 & \text { otherwise, }\end{cases}
$$

which converges to $E^{n}=W^{n}(X, L)$.

Proof. - Since $\operatorname{Supp}\left(D_{L} \mathcal{E}^{*}\right)=\operatorname{Supp}\left(\mathcal{E}^{*}\right)$, the filtration (29) is invariant under the derived duality. The decomposition of Proposition 7.1 is also invariant under the derived duality. So applying Theorem 3.1 and Proposition 7.1 gives a spectral sequence converging to $E^{n}=W^{n}(X, L)$ with terms

$$
E_{1}^{p q}=W^{p+q}\left(\mathbf{D}_{X}^{p} / \mathbf{D}_{X}^{p+1}, L\right)=\bigoplus_{x_{p} \in X^{(p)}} W^{p+q}\left(\mathbf{D}_{\mathrm{fl}}^{\mathrm{b}}\left(\mathrm{VB}_{\mathcal{O}_{x_{p}}}\right), L\right)
$$

if $0 \leqslant p \leqslant e$, while $E_{1}^{p q}=0$ for other values of $p$. Now over the spectrum of a local ring $\mathcal{O}_{x_{p}}$, the duality induced by $L$ and the 'usual' duality induced by $\mathcal{O}$ are (non-canonically) isomorphic. So we may apply Theorem 6.1 to see that

$$
W^{p+q}\left(\mathbf{D}_{\mathrm{fl}}^{\mathrm{b}}\left(\mathrm{VB}_{\mathcal{O}_{x_{p}}}\right), L\right) \cong \begin{cases}W\left(k\left(x_{p}\right)\right) & \text { if } p+q \equiv p(\bmod 4), \\ 0 & \text { otherwise }\end{cases}
$$

This completes the proof of the theorem.

Now consider an inclusion $i: U \hookrightarrow X$ with $U$ an open subscheme or with $U=\operatorname{Spec}\left(\mathcal{O}_{X, x}\right)$ where $\mathcal{O}_{X, x}$ is the local ring of a point of $X$. The restriction functor $i^{*}$ is exact and dualitypreserving and compatible with the filtrations

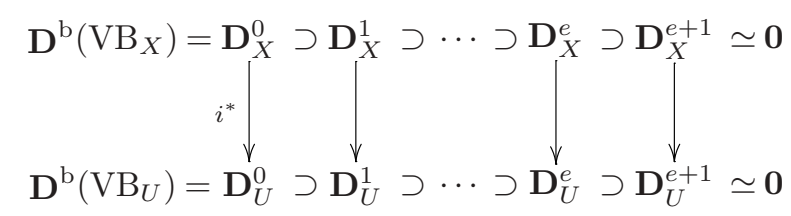

Applying Lemma 4.2 now gives the following result. 
LEMMA 7.3. - If $i: U \hookrightarrow X$ is as above, then $i^{*}$ induces a morphism of Gersten-Witt spectral sequences whose $E_{1}$-term is the natural restriction map of Gersten-Witt complexes

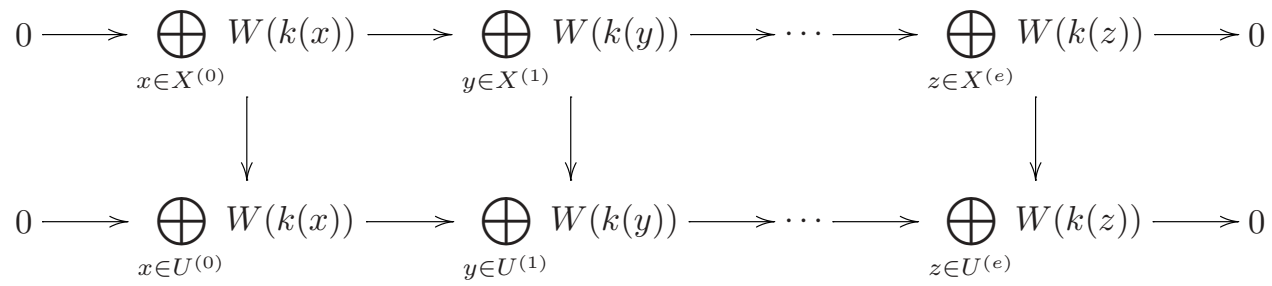

Remark. - Theorem 7.2 is also true for nonseparated $X$ which satisfy the other hypotheses of the theorem. In the proof, one replaces $\mathbf{D}^{\mathrm{b}}\left(\mathrm{VB}_{X}\right)$ by the category $\mathbf{D}_{\text {perf }}(X)$ of perfect complexes on $X$. The spectral sequence is unchanged except that it converges to the triangular Witt groups of $\mathbf{D}_{\text {perf }}(X)$.

If $X$ is noetherian and regular and has $\frac{1}{2} \in \Gamma\left(X, \mathcal{O}_{X}\right)$ but is of infinite Krull dimension, the Gersten-Witt complex and the spectral sequence exist, but we do not know whether the spectral sequence converges (see the discussion at the end of $\S 3$ ). Nagata's famous example of a noetherian ring of infinite Krull dimension is regular (see Eisenbud [7, Exercise 9.6]).

We hope to treat Gersten-Witt complexes on singular schemes in [4].

\section{More on the Gersten-Witt complex}

In this section we try to decorticate the coboundaries appearing on the first page of our spectral sequence and forming our Gersten-Witt complex. The reader will probably benefit from the comparison with the classical (second) residue homomorphism presented in Lemma 8.4.

We use the notations introduced at the beginning of Section 7. For simplicity we abbreviate $\mathbf{D}^{p}$ the subcategory of the derived category of $X$ on those complexes whose homology have support of codimension $\geqslant p$. Then the backbone of the Gersten-Witt complex is

$$
0 \rightarrow W^{0}\left(\mathbf{D}^{0} / \mathbf{D}^{1}\right) \rightarrow \cdots \rightarrow W^{p}\left(\mathbf{D}^{p} / \mathbf{D}^{p+1}\right) \rightarrow \cdots \rightarrow W^{e}\left(\mathbf{D}^{e}\right) \rightarrow 0
$$

obtained from the line $q=0$ in the general categorical spectral sequence of Theorem 3.1. The coboundary $d_{1}^{p 0}: W^{p}\left(\mathbf{D}^{p} / \mathbf{D}^{p+1}\right) \rightarrow W^{p+1}\left(\mathbf{D}^{p+1} / \mathbf{D}^{p+2}\right)$ is simply the composition

$$
W^{p}\left(\mathbf{D}^{p} / \mathbf{D}^{p+1}\right) \stackrel{\partial}{\longrightarrow} W^{p+1}\left(\mathbf{D}^{p+1}\right) \longrightarrow W^{p+1}\left(\mathbf{D}^{p+1} / \mathbf{D}^{p+2}\right)
$$

where the first map $\partial$ is the connecting homomorphism in the long exact sequence associated to $\mathbf{D}^{p+1} \hookrightarrow \mathbf{D}^{p} \rightarrow \mathbf{D}^{p} / \mathbf{D}^{p+1}$ as in Theorem 2.1, and where the second homomorphism is the natural localization.

On the backbone (31) we graft isomorphisms which identify $W^{p}\left(\mathbf{D}^{p} / \mathbf{D}^{p+1}\right)$ in local terms. These isomorphisms are those of Theorem 7.2 and they depend on choices of local parameters. Any two choices give isomorphic complexes, because they are both isomorphic to the above fundamental one. For instance, the exactness of the (augmented) Gersten-Witt complex is consequently independent of the choices.

The next part of the section will be spent showing that the differential $d_{1}^{00}: \mathcal{W}_{X, L}^{0} \rightarrow \mathcal{W}_{X, L}^{1}$ in our spectral sequence has the expected form. We first recall some definitions.

Let $A$ be a discrete valuation ring with residue field $k$, fraction field $F$, and uniformizing parameter $\pi$, and such that $\operatorname{char}(k) \neq 2$. Any quadratic space over $F$ is isometric to a diagonal 
space of the form $\left(F^{n+m}, \operatorname{diag}(\mathbf{a}, \pi \mathbf{b})\right)$ where $\mathbf{a}=\left(a_{1}, \ldots, a_{n}\right)$ and $\pi \mathbf{b}=\left(\pi b_{1}, \ldots, \pi b_{m}\right)$ with the $a_{i}, b_{j} \in A^{*}$. The first and second residue maps $W(F) \rightarrow W(k)$ are given by

$$
\begin{aligned}
& \operatorname{Res}_{A}^{1}\left(\left[F^{n+m}, \operatorname{diag}(\mathbf{a}, \pi \mathbf{b})\right]\right):=\left[k^{n}, \operatorname{diag}(\overline{\mathbf{a}})\right], \\
& \operatorname{Res}_{A}^{2}\left(\left[F^{n+m}, \operatorname{diag}(\mathbf{a}, \pi \mathbf{b})\right]\right):=\left[k^{m}, \operatorname{diag}(\overline{\mathbf{b}})\right]
\end{aligned}
$$

(cf. Milnor-Husemoller [14], Chap. IV (1.2)-(1.3)).

As a first step toward twisting Witt groups by line bundles, we study the twisted duality $D_{\pi^{r} A}:=\operatorname{Hom}_{A}\left(-, \pi^{r} A\right)$ on free $A$-modules. Thus let

$$
\mathbf{C}:=\mathbf{D}^{\mathrm{b}}\left(\mathrm{VB}_{A}\right), \quad \mathbf{D}:=\mathbf{D}_{\mathrm{fl}}^{\mathrm{b}}\left(\mathrm{VB}_{A}\right), \quad \mathbf{C} / \mathbf{D} \simeq \mathbf{D}^{\mathrm{b}}(F-\bmod ) .
$$

The quotient functor $\mathbf{C} \rightarrow \mathbf{C} / \mathbf{D}$ can be identified with the localization functor $-\otimes_{A} F$. If $\mathbf{C}$ and $\mathbf{D}$ are given the twisted duality $\left(D_{\pi^{r} A}, 1, \varpi_{\pi^{r} A}\right)$, then the induced duality on $\mathbf{C} / \mathbf{D}$ is $\operatorname{Hom}_{F}(-, F)$, and the morphism between the dualities on $\mathbf{C}$ and on $\mathbf{C} / \mathbf{D}$ comes from the inclusion $\pi^{r} A \subset F$.

LeMma 8.1. - If $\mathbf{C}$ and $\mathbf{D}$ are equipped with the twisted duality $D_{\pi^{r} A}=\operatorname{Hom}_{A}\left(-, \pi^{r} A\right)$, then the long exact sequence (11) of derived Witt groups

$$
\cdots \rightarrow W^{0}(\mathbf{C}) \rightarrow W^{0}(\mathbf{C} / \mathbf{D}) \rightarrow W^{1}(\mathbf{D}) \rightarrow \cdots
$$

reduces to the short exact sequence

$$
0 \rightarrow W\left(A, \pi^{r} A\right) \stackrel{i_{A, r}}{\longrightarrow} W(F) \stackrel{\partial_{A, r}}{\longrightarrow} W(k) \rightarrow 0
$$

where $i_{A, r}$ is the natural localization map and where $\partial_{A, r}=\operatorname{Res}_{A}^{2}$ if $r$ is even, while $\partial_{A, r}=\operatorname{Res}_{A}^{1}$ if $r$ is odd.

Proof. - The same argument as in the proof of Theorem 9.1 shows that (32) reduces to a short exact sequence of the form of (33). The inclusion map $W\left(A, \pi^{r} A\right) \rightarrow W(F)$ is clearly the localization map. We need to show that the map $\partial_{A, r}: W^{0}(\mathbf{C} / \mathbf{D}) \rightarrow W^{1}\left(\mathbf{D}, D_{\pi^{r} A}, 1, \varpi_{\pi^{r} A}\right)$ of derived Witt groups is given by $\partial_{A, r}=\operatorname{Res}_{A}^{1}$ if $r$ is odd and by $\partial_{A, r}=\operatorname{Res}_{A}^{2}$ if $r$ is even.

Give $\mathbf{D}=\mathbf{D}_{\mathrm{fl}}^{\mathrm{b}}\left(\mathrm{VB}_{A}\right)$ the unsigned shifted duality $\left(D_{1}^{\prime}, 1, \varpi_{\pi^{r} A}\right)$ of (25). The derived Witt group map is really a composition

$$
W^{0}(\mathbf{C} / \mathbf{D}) \stackrel{\eta}{\longrightarrow} W\left(\mathbf{D}, D_{1}^{\prime}, 1, \varpi_{\pi^{r} A}\right) \underset{\lambda}{\stackrel{\cong}{\leftrightarrows}} W^{0}\left(\mathbf{D}^{\mathrm{b}}(k-\bmod )\right),
$$

where $\eta$ is the composition of the coboundary of the long exact sequence (11) with the signchanging equivalence (25)-(26), and where $\lambda$ is induced by a functor $\mathbf{D}^{\mathrm{b}}(k$-mod) $\rightarrow \mathbf{D}$ sending

$$
[\cdots \rightarrow 0 \rightarrow 0 \rightarrow k \rightarrow 0 \rightarrow \cdots] \mapsto\left[\cdots \rightarrow 0 \rightarrow A \stackrel{\pi^{r+1}}{\longrightarrow} \pi^{r} A \rightarrow 0 \rightarrow \cdots\right]
$$

If $r=2 s$ is even, then $\eta$ sends

$$
\left[F^{n+m}, \operatorname{diag}(\mathbf{a}, \pi \mathbf{b})\right]=\left[F^{n+m}, \operatorname{diag}\left(\pi^{2 s} \mathbf{a}, \pi^{2 s+1} \mathbf{b}\right)\right]
$$


to the class of the symmetric complex

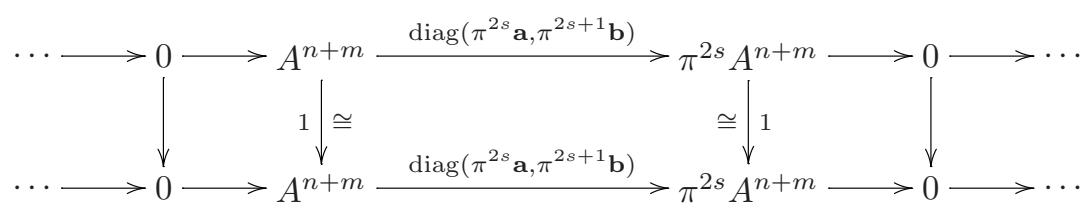

This symmetric complex is isometric in the derived category to

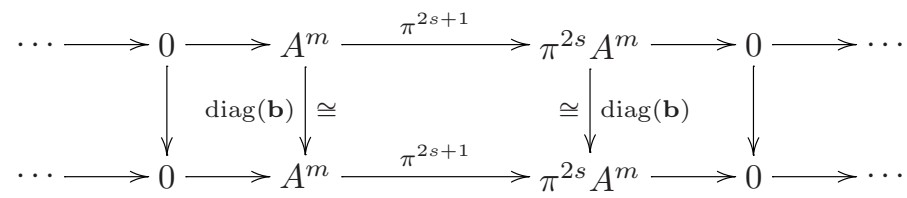

so its class is $\lambda\left(\left[k^{m}, \operatorname{diag}(\overline{\mathbf{b}})\right]\right)$. Thus the derived Witt group map $\partial_{A, r}: W(F) \rightarrow W(k)$ is the same as $\operatorname{Res}_{A}^{2}$ if $r$ is even. A similar calculation shows that $\partial_{A, r}=\operatorname{Res}_{A}^{1}$ if $r$ is odd.

Now let $X$ be a regular integral separated noetherian scheme with function field $K=K(X)$. A divisor on $X$ is a formal linear combination $E=\sum n_{x} x$ with the $x \in X^{(1)}$ and the $n_{x} \in \mathbb{Z}$ and all but finitely many $n_{x}=0$ (cf. Hartshorne [10, II.6]). For each $x \in X^{(1)}$ the local ring $\mathcal{O}_{X, x}$ is a discrete valuation ring with uniformizing parameter say $\pi_{x}$ and valuation $\operatorname{ord}_{x}$. Let $\partial_{x, n_{x}}: W(K) \rightarrow W(k(x))$ be as in Lemma 8.1, i.e.

$$
\partial_{x, n_{x}}= \begin{cases}\operatorname{Res}_{\mathcal{O}_{X, x}}^{2} & \text { if } n_{x} \text { is even } \\ \operatorname{Res}_{\mathcal{O}_{X, x}}^{1} & \text { if } n_{x} \text { is odd. }\end{cases}
$$

DEFINITION 8.2. - The unramified Witt group of $(X, E)$ is defined as

$$
W_{\mathrm{nr}}(X, E):=\operatorname{ker}\left(W(K) \stackrel{\left(\partial_{x, n_{x}}\right)}{\longrightarrow} \bigoplus_{x \in X^{(1)}} W(k(x))\right) .
$$

Associated to a divisor is a sheaf of fractional ideals $L=\mathcal{O}_{X}(-E) \subset K$ with fibers $L_{x}=\pi_{x}^{n_{x}} \mathcal{O}_{X, x} \subset K$. Since $X$ is regular, this sheaf of fractional ideals is invertible, i.e. is the sheaf of sections of a line bundle. Two divisors $E$ and $E_{1}$ correspond to isomorphic line bundles if and only if they are linearly equivalent, i.e. if and only if there exists $0 \neq g \in K$ such that $E_{1}=E+\operatorname{div}(g)$ where $\operatorname{div}(g):=\sum_{x} \operatorname{ord}_{x}(g) x$. Such linearly equivalent divisors generate a commutative diagram with exact rows and vertical isomorphisms

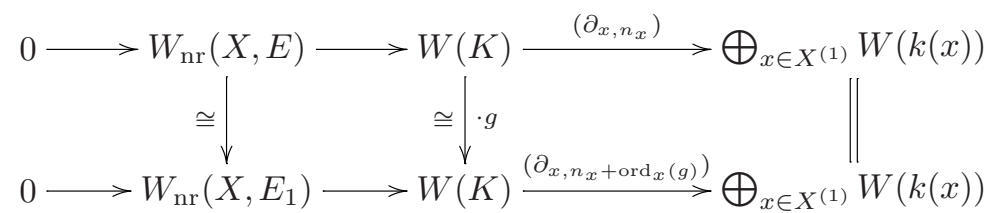

This allows us to define the unramified Witt group of a divisor class or of a line bundle $W_{\mathrm{nr}}(X, L)$, although to give an inclusion $W_{\mathrm{nr}}(X, L) \subset W(K)$ we must choose a divisor within the divisor class. 
If, as above, $L$ is a line bundle with fibers $L_{x}=\pi_{x}^{n_{x}} \mathcal{O}_{X, x}$, then for each $x$ the map $W(X, L) \rightarrow W(K)$ factors through $W\left(\mathcal{O}_{X, x}, L_{x}\right)=\operatorname{ker}\left(\partial_{x, n_{x}}\right)$, so there is a natural map $W(X, L) \rightarrow W_{\mathrm{nr}}(X, L)$.

DEFINITION 8.3. - One says that purity holds for the Witt groups of $(X, L)$ if the natural map $W(X, L) \rightarrow W_{\mathrm{nr}}(X, L)$ is an isomorphism, and we will say that weak purity holds if the map is surjective.

LEMMA 8.4. - Let $X$ and $L$ be as in Theorem 7.2.

(a) The residue map $\left(\partial_{x, n_{x}}\right)$ of (34) is the same as the differential $d_{1}: \mathcal{W}_{X, L}^{0} \rightarrow \mathcal{W}_{X, L}^{1}$ of our Gersten-Witt complex.

(b) The natural map $W(X, L) \rightarrow W_{\mathrm{nr}}(X, L)$ is the same as the map $W(X, L) \rightarrow H^{0}\left(\mathcal{W}_{X, L}\right)$ which is the spectral sequence edge map $E^{0} \rightarrow E_{2}^{00}$.

Proof. - (a) For any $x \in X^{(1)}$ the Gersten-Witt complex for $\left(\mathcal{O}_{X, x}, \pi_{x}^{n_{x}} \mathcal{O}_{X, x}\right)$ reduces to $0 \rightarrow W(K) \rightarrow W(k(x)) \rightarrow 0$. The differential is the residue map $\partial_{x, n_{x}}$ by Lemma 8.1. So if we project the Gersten-Witt complex of $X$ onto that of $\mathcal{O}_{X, x}$ using Lemma 7.3, we see that the general component $W(K) \rightarrow W(k(x))$ of the $d_{1}$-differential for $X$ is also $\partial_{x, n_{x}}$. This proves (a). Part (b) is left to the reader.

We now describe the components $\partial: W(k(x)) \rightarrow W(k(y))$ of the coboundary $d_{1}$ of the Gersten-Witt complex.

Suppose that $y$ is in $X^{(p)}$. If we apply Lemma 7.3 to $\operatorname{Spec}\left(\mathcal{O}_{X, y}\right) \subset X$, we see that $\partial$ factors through $\bigoplus_{z \in \operatorname{Spec}\left(\mathcal{O}_{X, y}\right)^{(p-1)}} W(k(z))$. So $\partial=0$ if $y \notin \overline{\{x\}}$. If $y \in \overline{\{x\}}$, then using the same lemma we reduce to $X=\operatorname{Spec}(\mathcal{O})$ with $\mathcal{O}$ a regular local ring of dimension $e=p$ whose maximal ideal $\mathfrak{m}$ corresponds to $y$ and with a prime ideal $\mathfrak{p} \subset \mathcal{O}$ of height $e-1$ corresponding to $x$. Then $A=\mathcal{O} / \mathfrak{p}$ is a Cohen-Macaulay local domain of Krull dimension 1 with dualizing module $\omega_{A}=\operatorname{Ext}_{\mathcal{O}}^{e-1}(A, \mathcal{O})$ and fraction field $k(x)$. There is a natural isomorphism $\widetilde{\varpi}: \operatorname{Ext}_{\mathcal{O}}^{e-1}\left(\omega_{A}, \mathcal{O}\right) \cong A$.

Now $\partial$ is really a coboundary $W\left(\mathcal{O}_{\mathfrak{p}}\right.$-fl-mod $) \rightarrow W(\mathcal{O}$-fl-mod $)$. Any class in $W\left(\mathcal{O}_{\mathfrak{p}}\right.$-fl-mod $)$ can be represented by a symmetric isomorphism $g: k(x)^{\oplus r} \cong \operatorname{Ext}_{\mathcal{O}_{\mathfrak{p}}}^{e-1}\left(k(x), \mathcal{O}_{\mathfrak{p}}\right)^{\oplus r}$. Replacing $g$ by an isometric map if necessary, we may assume that it lifts to a symmetric map $\bar{g}: A^{\oplus r} \rightarrow \omega_{A}^{\oplus r}$. There is then a commutative diagram with exact rows

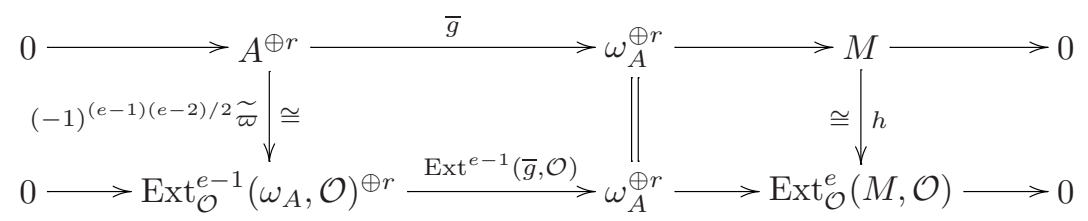

Comparing this diagram with the definition given for the coboundary of Theorem 2.1 and with diagram (9) completes the proof of the following proposition.

PROPOSITION 8.5. - Let $\partial: W(k(x)) \rightarrow W(k(y))$ be a component of the coboundary $d_{1}$ of the Gersten-Witt complex (1).

(a) If $y \notin \overline{\{x\}}$, then $\partial=0$.

(b) If $y \in \overline{\{x\}}$, then for any $\left[k(x)^{\oplus r}, g\right] \in W(k(x))$ the map $h$ defined above is a symmetric isomorphism, and $\partial\left(\left[k(x)^{\oplus r}, g\right]\right)=[M, h]$ in $W(\mathcal{O}$-fl-mod $) \cong W(k(y))$. 


\section{The punctured spectrum of a regular local ring}

We now describe the derived Witt groups of the punctured spectrum of a regular local ring. We will prove the following theorem, and then give some sufficient conditions for the splitting of the short exact sequence (36) appearing in the theorem.

THEOREM 9.1. - Let $\mathcal{O}$ be a regular local ring of dimension $e \geqslant 1$ with maximal ideal $\mathfrak{m}$ and residue field $k$. Suppose that $\operatorname{char}(k) \neq 2$. Let $U=\operatorname{Spec}(\mathcal{O}) \backslash\{\mathfrak{m}\}$ be the punctured spectrum. If $e \not \equiv 1(\bmod 4)$, then

$$
W^{n}(U) \cong \begin{cases}W(\mathcal{O}) & \text { if } n \equiv 0(\bmod 4) \\ W(k) & \text { if } n \equiv e-1(\bmod 4), \\ 0 & \text { otherwise. }\end{cases}
$$

If $e \equiv 1(\bmod 4)$, then there exists a short exact sequence

$$
0 \rightarrow W(\mathcal{O}) \rightarrow W(U) \rightarrow W(k) \rightarrow 0,
$$

while $W^{n}(U)=0$ for $n \not \equiv 0(\bmod 4)$.

All the identifications of Theorem 9.1 are isomorphisms or exact sequences of $W(\mathcal{O})$-modules because all the constructions used are compatible with tensoring with (the restriction of) a quadratic vector bundle on $\operatorname{Spec}(\mathcal{O})$.

Proof. - The triangulated category $\mathbf{C}:=\mathbf{D}^{\mathrm{b}}\left(\mathrm{VB}_{\mathcal{O}}\right)$ contains the saturated full triangulated subcategory $\mathbf{D}:=\mathbf{D}_{\mathrm{f}}^{\mathrm{b}}\left(\mathrm{VB}_{\mathcal{O}}\right)$, and the quotient triangulated category can be identified with $\mathbf{D}^{\mathrm{b}}\left(\mathrm{VB}_{U}\right)$ (see for example Balmer [3, Remark 4.9]). The long exact sequence (11) is then of the form

$$
\cdots \rightarrow W^{n}(\mathbf{D}) \rightarrow W^{n}(\mathbf{C}) \rightarrow W^{n}(U) \rightarrow W^{n+1}(\mathbf{D}) \rightarrow \cdots .
$$

Now by Theorem 6.1 one has $W^{n}(\mathbf{D})=W(k)$ if $n \equiv e(\bmod 4)$, and $W^{n}(\mathbf{D})=0$ otherwise. By Balmer [3, Theorem 5.6] one has $W^{m}(\mathbf{C})=W(\mathcal{O})$ if $m \equiv 0(\bmod 4)$, while $W^{m}(\mathbf{C})=0$ otherwise. So the long exact sequence (37) simplifies, but there are several cases to analyze.

If $e \equiv 1(\bmod 4)$, then the long exact sequence (37) degenerates into the short exact sequences

$$
0 \rightarrow W(\mathcal{O}) \rightarrow W^{4 \ell}(U) \rightarrow W(k) \rightarrow 0
$$

plus identifications $W^{n}(U)=0$ for $n \not \equiv 0(\bmod 4)$. If $e \equiv 2$ or $3(\bmod 4)$, then $(37)$ degenerates into isomorphisms

$$
W(\mathcal{O}) \stackrel{\cong}{\longrightarrow} W^{4 \ell}(U), \quad W^{4 \ell+e-1}(U) \stackrel{\cong}{\longrightarrow} W(k),
$$

plus identifications $W^{n}(U)=0$ for $n \not \equiv 0, e-1(\bmod 4)$. This proves the theorem for $e \not \equiv 0(\bmod 4)$.

If $e \equiv 0(\bmod 4)$, then one gets the same vanishings as in the immediately preceding case plus exact sequences

$$
0 \rightarrow W^{4 \ell-1}(U) \rightarrow W(k) \stackrel{\partial}{\longrightarrow} W(\mathcal{O}) \rightarrow W^{4 \ell}(U) \rightarrow 0 .
$$

It is enough to show that $\partial=0$. Now the restriction map $W(\mathcal{O}) \rightarrow W(k)$ is surjective and, as we said before beginning the proof of the theorem, it is a map of $W(\mathcal{O})$-modules. Similarly $\partial$ 
is a map of $W(\mathcal{O})$-modules. So for any $x \in W(\mathcal{O})$ we have $\partial(\bar{x})=x \cdot \partial(\overline{1})$. Now $\overline{1}$ is the class $[k,(1)]$, and it is sent to the minimal free resolution $\left[K, \eta_{k}\right]$ of (21). So it will be enough to show that $\partial(\overline{1})=\left[K, \eta_{k}\right]=0$ in $W^{e}(\mathcal{O})$ for even $e \geqslant 2$. The free module (21) can be written as $F=\mathcal{O} \oplus G$ with $G$ free of rank $e-1$. The module $\Lambda^{e / 2} F$ in the middle of (21) contains a Lagrangian submodule $\Lambda^{e / 2} G$, so the Koszul complex contains a Lagrangian subcomplex

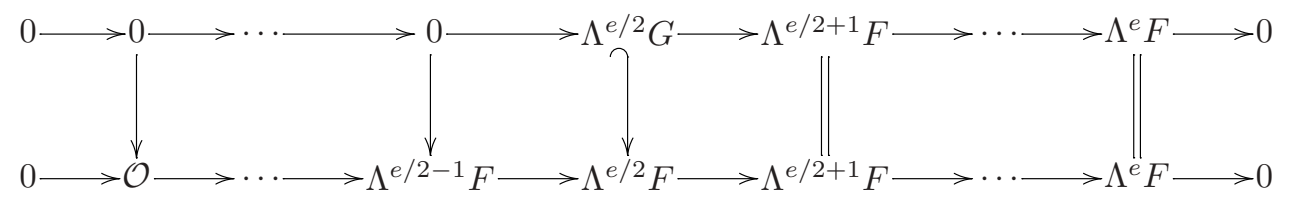

Therefore $\left[K, \eta_{k}\right]=0$, and $\partial=0$, completing the case $e \equiv 0(\bmod 4)$.

There are several situations in which we know that the exact sequence (36) splits:

Proposition 9.2. - Let $\mathcal{O}, k$, and $U$ be as in Theorem 9.1, and suppose that

$$
e=\operatorname{dim}(\mathcal{O}) \equiv 1(\bmod 4)
$$

Then the exact sequence (36) splits and $W(U) \cong W(\mathcal{O}) \oplus W(k)$ if any of the following holds: (a) if $\mathcal{O}$ contains a field and $e \geqslant 5$, (b) if $\mathcal{O}$ is complete or henselian, (c) if $\mathcal{O}$ is a k-algebra, or (d) if $\mathcal{O}=\mathbb{Z}_{(p)}$ with $p$ an odd prime and $U=\operatorname{Spec}(\mathbb{Q})$.

Proof. - (a) The natural map $W \rightarrow W_{\mathrm{nr}}$ from the Witt group to the unramified Witt group (described in (34) and Lemma 8.4(b) above) is functorial for inclusions of open subschemes. So we have a commutative diagram

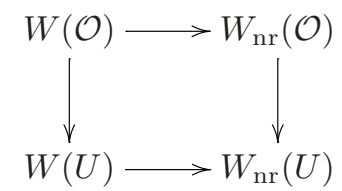

Since $e \geqslant 2$, the vertical map $W_{\mathrm{nr}}(\mathcal{O}) \rightarrow W_{\mathrm{nr}}(U)$ is an identity, since both groups are the kernel of $W(K) \rightarrow \bigoplus_{\mathrm{ht}(\mathfrak{p})=1} W(k(\mathfrak{p}))$, where $K$ is the field of fractions of $\mathcal{O}$. The horizontal map $W(\mathcal{O}) \rightarrow W_{\mathrm{nr}}(\mathcal{O})$ is an isomorphism by the Ojanguren-Panin purity theorem ([17, Theorem $\left.\left.\mathrm{A}\right]\right)$. Hence the vertical map $W(\mathcal{O}) \rightarrow W(U)$, which is the same as the inclusion map in the exact sequence (36), is retracted by the composition $W(U) \rightarrow W_{\mathrm{nr}}(U)=W_{\mathrm{nr}}(\mathcal{O}) \cong W(\mathcal{O})$. Moreover, all the maps are all compatible with tensoring with a quadratic $\mathcal{O}$-module, so the identification $W(U) \cong W(\mathcal{O}) \oplus W(k)$ is an isomorphism of $W(\mathcal{O})$-modules.

(b) It is well known that the natural restriction map $W(\mathcal{O}) \rightarrow W(k)$ is an isomorphism when $k$ is the residue field of a complete or henselian local ring. The exact sequence (36) is therefore an extension of free $W(k)$-modules and so is split. Thus $W(U) \cong W(k)^{\oplus 2}$, and the identification is an isomorphism of modules over $W(\mathcal{O})=W(k)$.

(c) The morphism $k \rightarrow \mathcal{O}$ defining the $k$-algebra induces a morphism $W(k) \rightarrow W(\mathcal{O})$ which makes (36) into an extension of $W(k)$-modules. Since the quotient module is projective, the extension splits. So there is a $W(k)$-linear isomorphism $W(U) \cong W(\mathcal{O}) \oplus W(k)$ in this case.

(d) This follows from Milnor-Husemoller [14] Chap. IV (2.1), where $W(\mathbb{Q})$ is calculated using a split exact sequence. 


\section{Purity and Gersten Conjecture in low dimensions}

In this section we prove our immediate applications of the Gersten-Witt spectral sequence, among which the Low Dimensions Theorem 10.1 and the Purity Corollaries 10.2 and 10.3. The notion of (weak) purity is recalled in Definition 8.3.

THEOREM 10.1 (Low dimensions). - Let $X$ and $L$ be as in Theorem 7.2. The derived Witt groups $W^{n}:=W^{n}(X, L)$ and the Witt cohomology groups $H^{n}(\mathcal{W}):=H^{n}\left(\mathcal{W}_{X, L}\right)$ have the following relationships:

(a) If $\operatorname{dim}(X) \leqslant 3$, then $W^{n} \cong H^{n}(\mathcal{W})$ for $0 \leqslant n \leqslant 3$.

(b) If $\operatorname{dim}(X)<n \leqslant 3$, then $W^{n}=0$.

(c) If $\operatorname{dim}(X) \leqslant 7$, then there is an exact sequence

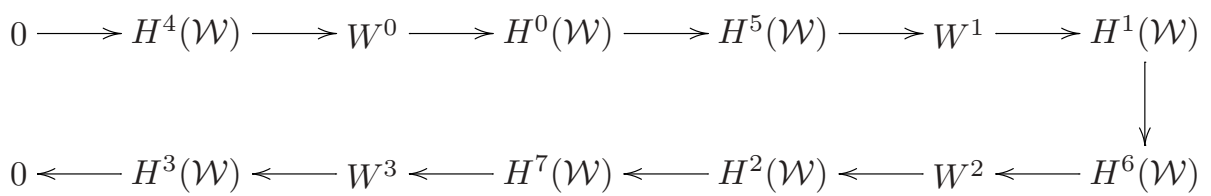

Proof. - (a) The only nonzero terms $E_{1}^{p q}$ are those with $q \equiv 0(\bmod 4)$ and with $0 \leqslant p \leqslant$ $\operatorname{dim}(X)$. So if $\operatorname{dim}(X) \leqslant 3$, and if $0 \leqslant n \leqslant 3$, then the only potentially nonzero term on the diagonal $p+q=n$ is $E_{1}^{n 0}=\mathcal{W}_{X, L}^{n}$, and the only potentially nonzero differentials going into or out of any $E_{r}^{n 0}$ are the $d_{1}$ differentials $\mathcal{W}_{X, L}^{n-1} \rightarrow \mathcal{W}_{X, L}^{n} \rightarrow \mathcal{W}_{X, L}^{n+1}$. So the abutment $E^{n}=W^{n}(X, L)$ is isomorphic to $E_{2}^{n 0}=H^{n}\left(\mathcal{W}_{X, L}\right)$.

(b) One has $W^{n}(X, L) \cong H^{n}\left(\mathcal{W}_{X, L}\right)$ by part (a), while $H^{n}\left(\mathcal{W}_{X, L}\right)=0$ because the GerstenWitt complex vanishes in dimensions $n>\operatorname{dim}(X)$.

(c) If $\operatorname{dim}(X) \leqslant 7$, and if $0 \leqslant n \leqslant 3$, then the only potentially nonzero terms on the diagonal $p+q=n$ are $E_{1}^{n 0}=\mathcal{W}_{X, L}^{n}$ and $E_{1}^{n+4,-4}=\mathcal{W}_{X, L}^{n+4}$, and the only potentially nonzero differentials are the $d_{1}$ and $d_{5}$. So the abutment $E^{n}=W^{n}(X, L)$ is an extension

$$
0 \rightarrow E_{6}^{n+4,-4} \rightarrow E^{n} \rightarrow E_{6}^{n 0} \rightarrow 0
$$

where

$$
\begin{aligned}
E_{6}^{n+4,-4} & =\operatorname{coker}\left(d_{5}: H^{n-1}\left(\mathcal{W}_{X, L}\right) \rightarrow H^{n+4}\left(\mathcal{W}_{X, L}\right)\right), \\
E_{6}^{n, 0} & =\operatorname{ker}\left(d_{5}: H^{n}\left(\mathcal{W}_{X, L}\right) \rightarrow H^{n+5}\left(\mathcal{W}_{X, L}\right)\right) .
\end{aligned}
$$

This can be put together to form the exact sequence of the theorem.

COROLlary 10.2. - Let $X$ and $L$ be as in Theorem 7.2. If $\operatorname{dim}(X)=4$, then weak purity holds for the Witt groups of $(X, L)$. More precisely, if $\operatorname{dim}(X) \leqslant 4$, the complex

$$
\bigoplus_{x \in X^{(3)}} W(k(x)) \rightarrow \bigoplus_{x \in X^{(4)}} W(k(x)) \rightarrow W(X, L) \rightarrow W(K) \rightarrow \bigoplus_{x \in X^{(1)}} W(k(x))
$$

is exact at the three middle groups.

Corollary 10.3. - Let $X$ and $L$ be as in Theorem 7.2. If $\operatorname{dim}(X) \leqslant 3$, then purity holds for the Witt groups of $(X, L)$.

Proof. - Those corollaries follow from our identifying of $d_{1}^{00}: \mathcal{W}_{X, L}^{0} \rightarrow \mathcal{W}_{X, L}^{1}$ as a 'twisted' residue map (Lemma 8.4), thus obtaining $H^{0}\left(\mathcal{W}_{X, L}\right)=W_{\mathrm{nr}}(X, L)$. Using then Theorem 10.1, 
the definition of $H^{4}\left(\mathcal{W}_{X, L}\right)$ and the observation that $H^{5}\left(\mathcal{W}_{X, L}\right)=0$ when $\operatorname{dim}(X) \leqslant 4$ leads immediately to the result.

Another application of Theorem 10.1 is the following result.

COROLlaRY 10.4. - Let $X=\operatorname{Spec}(R)$ for $R$ a regular local ring of Krull dimension e $\leqslant 4$ and containing $\frac{1}{2}$. Then the Gersten Conjecture holds, namely the augmented Gersten-Witt complex (4) is exact.

Proof. - Let us identify as many groups as we can in the exact sequence of Theorem 10.1. First of all, observe that the dimension hypothesis forces $H^{5}(\mathcal{W})=H^{6}(\mathcal{W})=H^{7}(\mathcal{W})=0$. Recall from Balmer [3, Theorem 5.6], that $W^{1}(R)=W^{2}(R)=W^{3}(R)=0$. We also have $H^{4}(\mathcal{W})=0$. This is obvious if $e=\operatorname{dim}(R)<4$. If $e=4$, observe that we always have $H^{\operatorname{dim} R}(\mathcal{W})=0$. In fact, if $\mathcal{O}$ is a discrete valuation ring, the second residue $W(K) \rightarrow W(k)$ is obviously surjective. Now, let $x_{1}, \ldots, x_{e}$ be a system of regular parameters of $R$, then $R /\left(x_{2}, \ldots, x_{e}\right)$ is a discrete valuation ring and one deduces immediately $H^{\operatorname{dim} R}(\mathcal{W})=0$ from Proposition 8.5.

Now, we deduce from the exact sequence of Theorem 10.1, that

$$
H^{1}(\mathcal{W})=H^{2}(\mathcal{W})=H^{3}(\mathcal{W})=0
$$

and that $H^{0}(\mathcal{W})=W(R)$. This is the claim.

\section{Concluding remarks}

The special significance of dimension 4 for the purity of Witt groups has been observable for some time. For instance, when $R$ is a regular domain with fraction field $K$ and $\frac{1}{2} \in R$, then it has been known that the map $W(R) \rightarrow W(K)$ is injective if $\operatorname{dim}(R) \leqslant 3$ but is not always injective if $\operatorname{dim}(R)=4$. The Gersten-Witt spectral sequence explains this 'dimension gap' as a consequence of the vanishing of three out of every four lines of the spectral sequence, with the effect that the coniveau filtration of the Witt group $W(R)$ has contributions only from codimensions $0,4,8, \ldots$ At a deeper level, dévissage reduces the computation of the spectral sequence to the derived Witt groups of fields, and three out of four derived Witt groups of a field vanish, while every fourth one is the usual Witt group $W^{4 \ell}(k)=W(k)$.

Thus the special geography of the Gersten-Witt spectral sequence gives a convincing reason why purity holds until a first gap after dimension 3, and it brings to light the long exact sequence of Theorem 10.1(c), which works until a second gap after dimension 7 . The spectral sequence also reveals that 'weak purity' holds at the first boundary dimension 4, but we have no idea how long that phenomenon goes on.

\section{Acknowledgement}

We would like to thank Bruno Kahn for telling us we could simplify our approach by using exact couples. We sincerely thank Stefan Gille for his careful reading of a preliminary version. The first author is grateful to the University of Nice for inviting him and offering the nice environment which led to the present article. He has also been supported by NSERC and the University of Western Ontario. The second author would like to thank Manuel Ojanguren and the University of Lausanne for several invitations.

\section{REFERENCES}

[1] Balmer P., Derived Witt groups of a scheme, J. Pure Appl. Algebra 141 (1999) 101-129. 
[2] Balmer P., Triangular Witt groups. Part I: The 12-term localization exact sequence, K-Theory 19 (2000) 311-363.

[3] Balmer P., Triangular Witt groups. Part II: From usual to derived, Math. Z. 236 (2001) 351-382.

[4] Balmer P., Walter C., Derived Witt groups and Grothendieck duality, in preparation.

[5] Beilinson A., Bernstein J., Deligne P., Faisceaux pervers, Astérisque 100 (1982).

[6] Cartan H., Eilenberg S., Homological Algebra, Princeton Univ. Press, 1956.

[7] EISEnbud D., Commutative Algebra with a View Toward Algebraic Geometry, Springer-Verlag, 1995.

[8] ETTNER A., Zur Residuenabbildung in der Theorie quadratischer Formen, Diplomarbeit, Regensburg, 1999.

[9] FernándeZ-CARMEnA F., On the injectivity of the map of the Witt group of a scheme into the Witt group of its function field, Math. Ann. 277 (1987) 453-468.

[10] Hartshorne R., Algebraic Geometry, Springer-Verlag, 1977.

[11] Keller B., On the cyclic homology of exact categories, J. Pure Appl. Algebra 136 (1999) 1-56.

[12] Keller B., Appendix: On Gabriel-Roiter's axioms for exact categories, Trans. Amer. Math. Soc. 351 (1999) 677-681.

[13] Mac Lane S., Categories for the Working Mathematician, 2nd edition, Springer-Verlag, 1998.

[14] Milnor J., Husemoller D., Symmetric Bilinear Forms, Springer-Verlag, 1973.

[15] NeEman A., The derived category of an exact category, J. Algebra 135 (1990) 388-394.

[16] Ojanguren M., Parimala R., Sridharan R., Suresh V., Witt groups of the punctured spectrum of a 3-dimensional local ring and a purity theorem, J. London Math. Soc. 59 (1999) 521-540.

[17] Ojanguren M., Panin I., A purity theorem for the Witt group, Ann. Scient. Éc. Norm. Sup. (4) 32 (1999) 71-86.

[18] PARdon W., A relation between Witt groups and 0-cycles in a regular ring, in: Springer Lect. Notes Math., Vol. 1046, 1984, pp. 261-328.

[19] PARDon W., The filtered Gersten-Witt resolution for regular schemes, Preprint, 2000, http://www. math.uiuc.edu/K-theory/0419/.

[20] Parimala R., Witt groups of affine three-folds, Duke Math. J. 57 (1988) 947-954.

[21] Quebbemann H.-G., Scharlau W., Schulte M., Quadratic and Hermitian forms in additive and Abelian categories, J. Algebra 59 (1979) 264-289.

[22] Ranicki A., Algebraic L-theory. I. Foundations, Proc. London Math. Soc. (3) 27 (1973) 101-125.

[23] Ranicki A., Additive $L$-theory, K-Theory 3 (1989) 163-195.

[24] Rost M., http://www.math.ohio-state.edu/ rost/schmid.html.

[25] SCHMid M., Wittringhomologie, Ph.D. dissertation, Regensburg 1997. Cf. [24].

[26] Verdier J.-L., Des catégories dérivées des catégories abéliennes (Thèse de doctorat d'état, Paris, 1967), Astérisque 239 (1996).

[27] Walter C., Obstructions to the Existence of Symmetric Resolutions, in preparation.

[28] Weibel C., An Introduction to Homological Algebra, Cambridge Univ. Press, 1994.

(Manuscript received April 10, 2000; accepted, after revision, January 26, 2001.)

Paul BALMER

SFB 478, Universität Münster,

Hittorfstr. 27,

48149 Münster, Germany

E-mail: balmer@math.uni-muenster.de

Charles WALTER

Laboratoire J.-A. Dieudonné

(UMR $6621 \mathrm{du}$ CNRS),

Université de Nice - Sophia Antipolis,

06108 Nice Cedex 02, France

E-mail: walter@math.unice.fr 